\title{
Forty-four New and Known M-dwarf Multiples in the SDSS-III/APOGEE M-dwarf Ancillary Science Sample
}

\author{
Jacob Skinner ${ }^{1}$, Kevin R. Covey ${ }^{1}\left(\mathbb{D}\right.$, Chad F. Bender $^{2}(\mathbb{D})$, Noah Rivera ${ }^{2}$, Nathan De Lee ${ }^{3,4}\left(\mathbb{D}\right.$, Diogo Souto $^{5}$, Drew Chojnowski ${ }^{6}$, \\ Nicholas Troup $^{7}$, Carles Badenes ${ }^{8}$ (i), Dmitry Bizyaev ${ }^{6}$ (i) , Cullen H. Blake $^{9}$ (i), Adam Burgasser ${ }^{10}$ (i), Caleb Cañas ${ }^{11}$, \\ Joleen Carlberg $^{12}$ (1) , Yilen Gómez Maqueo Chew ${ }^{13}$, Rohit Deshpande ${ }^{11}$, Scott W. Fleming ${ }^{12}$ (1) J. G. Fernández-Trincado ${ }^{14,15}$, \\ D. A. García-Hernández ${ }^{16,17}$ (1), Fred Hearty ${ }^{11}$, Marina Kounkel ${ }^{1}$ (iD), Penélope Longa-Peñe ${ }^{18}$, Suvrath Mahadevan ${ }^{11}{ }^{(1)}$, \\ Steven R. Majewski ${ }^{19}$ (1), Dante Minniti ${ }^{20}$ (1), David Nidever ${ }^{21}$ (1), Audrey Oravetz ${ }^{6}$, Kaike $\operatorname{Pan}^{6}$ (10), Keivan Stassun ${ }^{22}$ (1), \\ Ryan Terrien ${ }^{23}$, and Olga Zamora ${ }^{16,17}$ \\ ${ }^{1}$ Department of Physics \& Astronomy, Western Washington University, Bellingham, WA 98225, USA; skinnej3@wwu.edu, kevin.covey@wwu.edu \\ ${ }_{3}^{2}$ Department of Astronomy and Steward Observatory, University of Arizona, Tucson, AZ 85721, USA; cbender@email.arizona.edu \\ ${ }^{3}$ Department of Physics, Geology, and Engineering Technology, Northern Kentucky University, Highland Heights, KY 41099, USA \\ ${ }^{4}$ Department of Physics \& Astronomy, Vanderbilt University, Nashville, TN 37235, USA \\ ${ }_{6}^{5}$ Observatório Nacional, Rua General José Cristino, 77, 20921-400 São Cristóvão, Rio de Janeiro, RJ, Brazil \\ ${ }^{6}$ Apache Point Observatory and New Mexico State University, P.O. Box 59, Sunspot, NM 88349-0059, USA \\ ${ }^{7}$ Department of Physics, Salisbury University, 1101 Camden Ave., Salisbury, MD 21801, USA \\ ${ }^{8}$ Department of Physics and Astronomy, University of Pittsburgh, Allen Hall, 3941 O'Hara St., Pittsburgh, PA 15260, USA \\ ${ }^{9}$ Department of Physics and Astronomy, University of Pennsylvania, 209 South 33rd Street, Philadelphia, PA 19104, USA \\ ${ }^{10}$ Center for Astrophysics and Space Science, University of California San Diego, La Jolla, CA 92093, USA \\ ${ }^{11}$ Department of Astronomy \& Astrophysics, Pennsylvania State, 525 Davey Lab, University Park, PA 16802, USA \\ ${ }^{12}$ Space Telescope Science Institute, Baltimore, MD 21218, USA \\ ${ }^{13}$ Instituto de Astronomía, Universidad Nacional Autónoma de México, Ciudad Universitaria, Ciudad de México, 04510, México \\ ${ }^{14}$ Departamento de Astronomía, Casilla 160-C, Universidad de Concepción, Concepción, Chile \\ ${ }^{15}$ Institut Utinam, CNRS UMR6213, Univ. Bourgogne Franche-Comté, OSU THETA, Observatoire de Besançon, BP 1615, F-25010 Besançon Cedex, France \\ ${ }^{16}$ Instituto de Astrofísica de Canarias (IAC), Vía Lactea s/n, E-38205 La Laguna, Tenerife, Spain \\ ${ }^{17}$ Departamento de Astrofísica, Universidad de La Laguna (ULL), E-38206 La Laguna, Tenerife, Spain \\ ${ }^{18}$ Unidad de Astronomía, Facultad de Ciencias Básicas, Avenida Angamos 601, Antofagasta 1270300, Chile \\ ${ }^{19}$ Department of Astronomy, University of Virginia, Charlottesville, VA 22904-4325, USA \\ ${ }^{20}$ Pontificia Universidad Católica de Chile, Instituto de Astrofsica, Av. Vicuna Mackenna 4860, 782-0436 Macul, Santiago, Chile \\ ${ }^{21}$ Department of Physics, Montana State University, Bozeman, MT 59717, USA \\ ${ }^{22}$ Department of Physics and Astronomy, Vanderbilt University, VU Station 1807, Nashville, TN 37235, USA \\ ${ }^{23}$ Department of Physics \& Astronomy, Carleton College, Northfield, MN 55057, USA \\ Received 2018 February 13; revised 2018 May 29; accepted 2018 May 30; published 2018 July 6
}

\begin{abstract}
Binary stars make up a significant portion of all stellar systems. Consequently, an understanding of the bulk properties of binary stars is necessary for a full picture of star formation. Binary surveys indicate that both multiplicity fraction and typical orbital separation increase as functions of primary mass. Correlations with higherorder architectural parameters such as mass ratio are less well constrained. We seek to identify and characterize double-lined spectroscopic binaries (SB2s) among the 1350 M-dwarf ancillary science targets with APOGEE spectra in the SDSS-III Data Release 13. We measure the degree of asymmetry in the APOGEE pipeline crosscorrelation functions (CCFs) and use those metrics to identify a sample of 44 high-likelihood candidate SB2s. At least 11 of these SB2s are known, having been previously identified by Deshpande et al. and/or El-Badry et al. We are able to extract radial velocities (RVs) for the components of 36 of these systems from their CCFs. With these RVs, we measure mass ratios for 29 SB2s and five SB3s. We use Bayesian techniques to fit maximum-likelihood (but still preliminary) orbits for four SB2s with eight or more distinct APOGEE observations. The observed (but incomplete) mass-ratio distribution of this sample rises quickly toward unity. Two-sided Kolmogorov-Smirnov tests find probabilities of $18.3 \%$ and $18.7 \%$, demonstrating that the mass-ratio distribution of our sample is consistent with those measured by Pourbaix et al. and Fernandez et al., respectively.
\end{abstract}

Key words: binaries: close - binaries: general - binaries: spectroscopic - stars: formation - stars: low-mass

Supporting material: machine-readable tables

\section{Introduction}

Models of stellar formation and evolution make predictions about the distribution and frequency of stellar binaries. Fragmentation of a protostellar core or circumstellar disk can produce the requisite pair of pre-main-sequence stars (e.g., Offner et al. 2010), but only at much larger separations $(\sim 100-1000+$ au; Tohline 2002; Kratter 2011) than those that characterize $\operatorname{close}^{24}$

\footnotetext{
${ }^{24}$ With separations on the order of $\sim 1$ au, i.e., noninteracting and spectroscopic.
}

binaries. Dynamical processes presumably drive some of these wider binaries into a close configuration, but the nature and timescale of this evolution remains unclear: mechanisms that may play a role include dynamical friction from gas in the surrounding disk or core (e.g., Gorti \& Bhatt 1996), gravitational interactions in/dynamical decay of few-body systems (e.g., Reipurth \& Clarke 2001; Bate et al. 2002), Kozai oscillations (Fabrycky \& Tremaine 2007), and/or tidal friction (e.g., Kiseleva et al. 1998). 
Empirical study has provided some data with which to test these models. The multiplicity fraction (MF; $\frac{\# \text { multiples }}{\text { all stars }}$ ) is known to be an increasing function of primary mass: the lowest multiplicity rates are observed for substellar systems $(\mathrm{MF}$ $11_{-2}^{+7} \%$, implying a companion fraction $(\mathrm{CF}) \frac{\# \text { stars with companions }}{\text { all stars }}$ of $\approx 20 \%$; Burgasser et al. 2006) and rise into the M-dwarf regime, where the seminal measurement of the $\mathrm{CF}$ over all separations remains that of Fischer \& Marcy (1992; hereafter FM1992): $42 \% \pm 9 \%$ for separations of $0.04-10^{4}$ au. Yet larger multiplicity rates are found for stars of G-type $(46 \% \pm$ 2\%; Raghavan et al. 2010) and F-type and earlier (100\% \pm 20\%; Duchêne \& Kraus 2013). There is also mounting evidence of a trend of binary separation increasing with primary mass (Ward-Duong et al. 2015). When corrected for incompleteness, the mass-ratio distribution of close binaries is mostly flat (Moe \& Di Stefano 2017).

A particularly common result of the star formation process, M dwarfs, by virtue of their low masses, provide leverage for probing the link between primary mass, CF, and orbital separation. Since the survey of FM1992, additional M-dwarf multiplicity surveys have been conducted by Clark et al. (2012; hereafter CBK2012), Shan et al. (2015), and Ward-Duong et al. (2015), who used various observational techniques to identify 22,12 , and 65 multiple systems within samples of 1452, 150, and $245 \mathrm{M}$ dwarfs, respectively. These measurements are consistent with the FM1992 result, suggesting a CF of 26\%$35 \%$ for separations outside a key gap in coverage from 0.4 to $3 \mathrm{au}$. The near-infrared spectra of the APOGEE survey are well suited to detect the faint, cool companions of $\mathrm{M}$ dwarfs. This gives us a window into the dynamic evolution of early systems, as well as developed systems in the low-period regime. A survey of M-dwarf double-lined spectroscopic binaries (SB2s) in clusters and the field could detect changes in the close binary fraction with age, providing a valuable clue as to whether lowperiod binaries most often mutually form up close or evolve through three-body dynamics with a third, distant companion.

In this paper, we search the APOGEE spectroscopic database for close, double-lined SB2s with low-mass M-dwarf primaries. We utilize a classic approach, searching for sources whose spectra include two or more sets of photospheric absorption lines with a clear radial velocity (RV) offset in at least one APOGEE observation. This approach compliments the recent search conducted by El-Badry et al. (2018b), using the direct spectral modeling approach validated by El-Badry et al. (2018a). The search completed by El-Badry et al. (2018b) is sensitive to multiple systems over a much larger range of orbital separations, as their method can detect spectral superpositions even with no RV offset. While their search is sensitive to a much broader range of parameter space in the dimension of orbital separation, their spectral modeling approach is limited to stars with $T_{\text {eff }}>4000 \mathrm{~K}$, providing motivation for a directed search for close, low-mass SB2s.

We begin by introducing the observational data and describing our sample selection in Section 2. We describe our data analysis procedure, mass-ratio measurements, and mass-ratio distribution in Section 3. Section 4 contains the description of our orbit-fitting procedure and results for four targets. Finally, we present our results in Section 5 and summarize our conclusions in Section 6. The Appendix contains notes on a mass estimation calculation mentioned in Section 4.2.

\section{Observations and Sample Selection}

\subsection{SDSS-III APOGEE M-dwarf Ancillary Targets}

The SDSS-III (Eisenstein et al. 2011) APOGEE M-dwarf ancillary program (Deshpande et al. 2013; Holtzman et al. 2015) was designed to produce a large, homogeneous spectral library and kinematic catalog of nearby low-mass stars; these data products are useful for investigations of stellar astrophysics (e.g., Souto et al. 2017; Gilhool et al. 2018) and refining targeting procedures for current and future exoplanet search programs. These science goals are uniquely enabled by the APOGEE spectrograph (Wilson et al. 2010, 2012), which acquires highresolution $(R \sim 22,000)$ near-infrared spectra from each of 300 optical fibers. As deployed at the $2.5 \mathrm{~m}$ SDSS telescope (Gunn et al. 2006), the APOGEE spectrograph achieves a field of view with a diameter of $3^{\circ}$, making it a highly efficient instrument for surveying the stellar parameters of the constituents of Galactic stellar populations (Majewski et al. 2017). The SDSS DR13 data release (Albareti et al. 2017) includes 7152 APOGEE spectra of 1350 stars targeted by this ancillary program. The methods used to select targets for the SDSS ancillary program are described in full by Deshpande et al. (2013) and Zasowski et al. (2013); briefly, the targets were selected with one of the following methods.

1. Stars of spectral type M4 or later, typically toward the fainter end of APOGEE's sensitivity range $(H \gtrsim 10)$, were targeted by applying a set of magnitude $(7<H<12)$ and color $\left(V-K_{s}>5.0 ; 0.4<J-H<0.65 ; 0.1<H-\right.$ $\left.K_{s}<0.42\right)$ cuts to the catalog of northern high-proper-

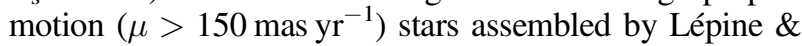
Shara (2005).

2. The M dwarfs of all spectral subclasses, typically toward the brighter end of APOGEE's sensitivity range $(H \lesssim 10)$, were identified by applying simple spatial (decl. $>0$ ) and magnitude $(H>7)$ cuts to the all-sky catalog of bright $\mathrm{M}$ dwarfs assembled by Lépine \& Gaidos (2011).

3. Calibrators with precise, stable RVs (as measured by the California Planet Search team); reliable measurements of rotation velocity (Jenkins et al. 2009; $v \sin i$ ); active M dwarfs in the Kepler field (Ciardi et al. 2011; Walkowicz et al. 2011); or targets in the input catalog of the MEarth Project (Nutzman \& Charbonneau 2008) were individually added to the sample.

Figure 1 shows the location of these 1350 ancillary targets in $J-H$ versus $H-K_{s}$ color-color space, along with the full DR13 sample shown for context. Figure 2 compares the number of APOGEE observations obtained for objects identified here as binaries relative to the number of observations obtained for the full DR13 sample and the subset of M-dwarf ancillary science targets. On average, sources identified as SB2s have one more APOGEE observation than the median for the M-dwarf ancillary science sample, reflecting the advantage that multi-epoch observations provide for identifying RV variable sources.

\subsection{Identification as $S B 2$ s}

Candidate SB2s were identified with an approach similar to that of Fernandez et al. (2017; hereafter F17), who flagged sources with significant asymmetries in the cross-correlation functions (CCFs) calculated by the APOGEE pipeline (Nidever et al. 2015; García Pérez et al. 2016; Grieves et al. 2017). 


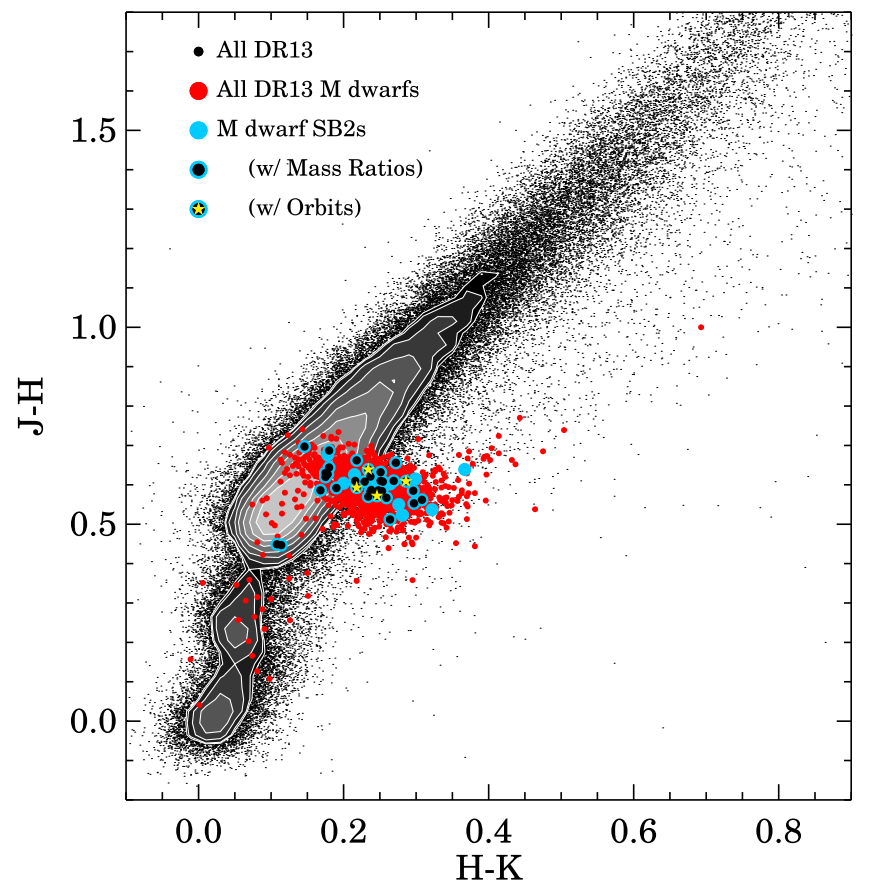

Figure 1. $J-H$ vs. $H-K_{s}$ color-color diagram of DR13 APOGEE targets. The full DR13 sample is shown as small points and gray-scale contours in areas of color space where individual points can no longer be distinguished. The M-dwarf ancillary targets are shown as red dots, demonstrating the clear divergence from the reddened giant branch that makes up the bulk of the APOGEE data set. Candidate SB2s are indicated with cyan dots; sources for which we infer mass ratios and full orbital fits are highlighted with a black central dot and surrounding ring, respectively.

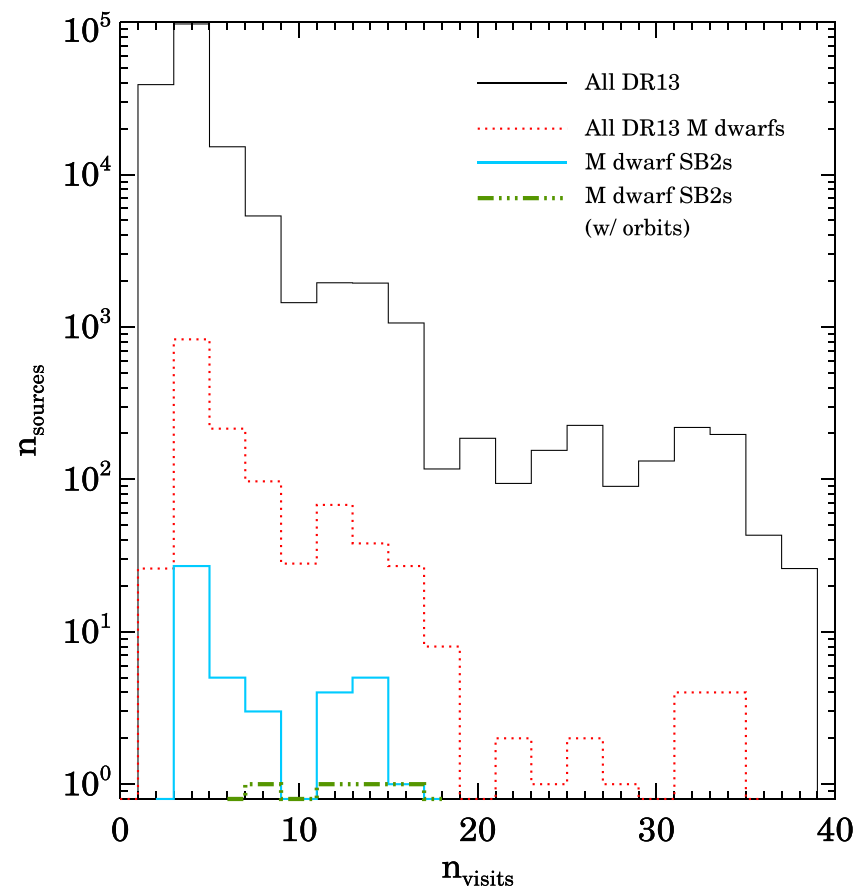

Figure 2. Histograms of the number of visits observed for different classes of APOGEE DR13 targets. The APOGEE M-dwarf sample exhibits the same overall distribution of visits as the rest of the survey targets; the M-dwarf SB2 candidates are modestly biased toward a larger number of visits, with $\sim$ one more visit per system in both the median and the mean than the broader DR13 sample.
Following F17, we characterized the asymmetry in each $\mathrm{CCF}$ using the $R$ parameter originally developed by Tonry \& Davis (1979),

$$
R=\frac{H}{\sqrt{2} \sigma_{a}},
$$

where $H$ is the maximum of the CCF, and $\sigma_{a}$ is the rms of the antisymmetric portion of the CCF. In this formalism, lower $R$ values indicate sources with larger asymmetries in their $\mathrm{CCF}$ functions. To better identify sources with CCF asymmetries at physically meaningful velocity separations, we computed distinct $R$ values for windows of differing widths around each CCF's central peak. Specifically, we computed $R$ values for the central 51, 101, and 151 lags in each CCF, which we denote as $R_{51}, R_{101}$, and $R_{151}$, respectively. Given the $4.14 \mathrm{~km} \mathrm{~s}^{-1}$ pixel spacing of the APOGEE spectra, these CCF windows provide sensitivity to secondaries with velocity separations from the primary star of 106,212 , and $318 \mathrm{~km} \mathrm{~s}^{-1}$.

We used a combination of absolute and relative criteria to identify candidate SB2s based on the lowest $R$ values they exhibited across all their APOGEE observations. Selecting candidates on the basis of their lowest observed $R$ values allows us to identify systems even if they only exhibit a clear velocity separation in a single epoch of APOGEE spectra. Absolute criteria ensure that each star's CCF exhibits an asymmetry substantial enough to indicate the presence of a secondary star, while relative criteria based on ratios of the $R$ values measured from different portions of the $\mathrm{CCF}$ (e.g., $R_{51}, R_{151}$, etc.) eliminate false positives due to sources whose CCFs exhibit significant asymmetries but at velocities too large to be physically plausible for a bona fide SB2. We denote the smallest $R$ value observed within a given CCF window across all of a star's APOGEE observations as $\min _{R_{W}}$ (where $W$ indicates the width of the CCF window the $R$ value was computed from, such that $\min _{R_{151}}$ indicates the smallest $R_{151}$ observed for a given star). To provide additional measures of the structure of each $\mathrm{CCF}$, we also record the maximum response and bisector width of each $\mathrm{CCF}$ as peak and bisector $_{X}$, respectively. Following the notation for the minimum $R$ values across all epochs, we denote the maximum $\mathrm{CCF}$ response and bisector width across all observations as $\max _{\text {bisector }_{x}}$ and $\max _{\text {peak }}$, respectively. Using these measures of the structure detected across all CCFs computed for a given source, we identify candidate SB2s with the following criteria.

1. To identify sources that exhibit a strong central asymmetry on at least one epoch, we require

$$
-\log _{10}\left(\min _{R_{101}}\right)<0.83 \text { AND } 0.06<\log 10 \frac{\min _{R_{151}}}{\min _{R_{101}}}<0.13
$$
OR

$-\log _{10}\left(\min _{R_{51}}\right)<0.83$ AND $0.05<\log _{10} \frac{\min _{R_{101}}}{\min _{R_{51}}}<0.2$.

2. To eliminate sources with weak CCF responses, suggesting a poor template match, we require $-\log _{10}\left(\max _{\text {peak }}\right)>-0.5$.

3. To eliminate sources whose CCF peaks are indicative of very low $\mathrm{S} / \mathrm{N}$ or a reduction issue (i.e., too narrow or wide to be consistent with a single star or binary or containing a greater degree of asymmetry than expected for two to three well-detected CCF peaks), we require

$-0.7>\log _{10}\left(\max _{\text {bisector }_{x}}\right)>2.3$

$-\log _{10}\left(\min _{R_{51}}\right)>0.25$

$-\log _{10}\left(\min _{R_{101}}\right)>0.22$. 
Table 1

Selected Binaries

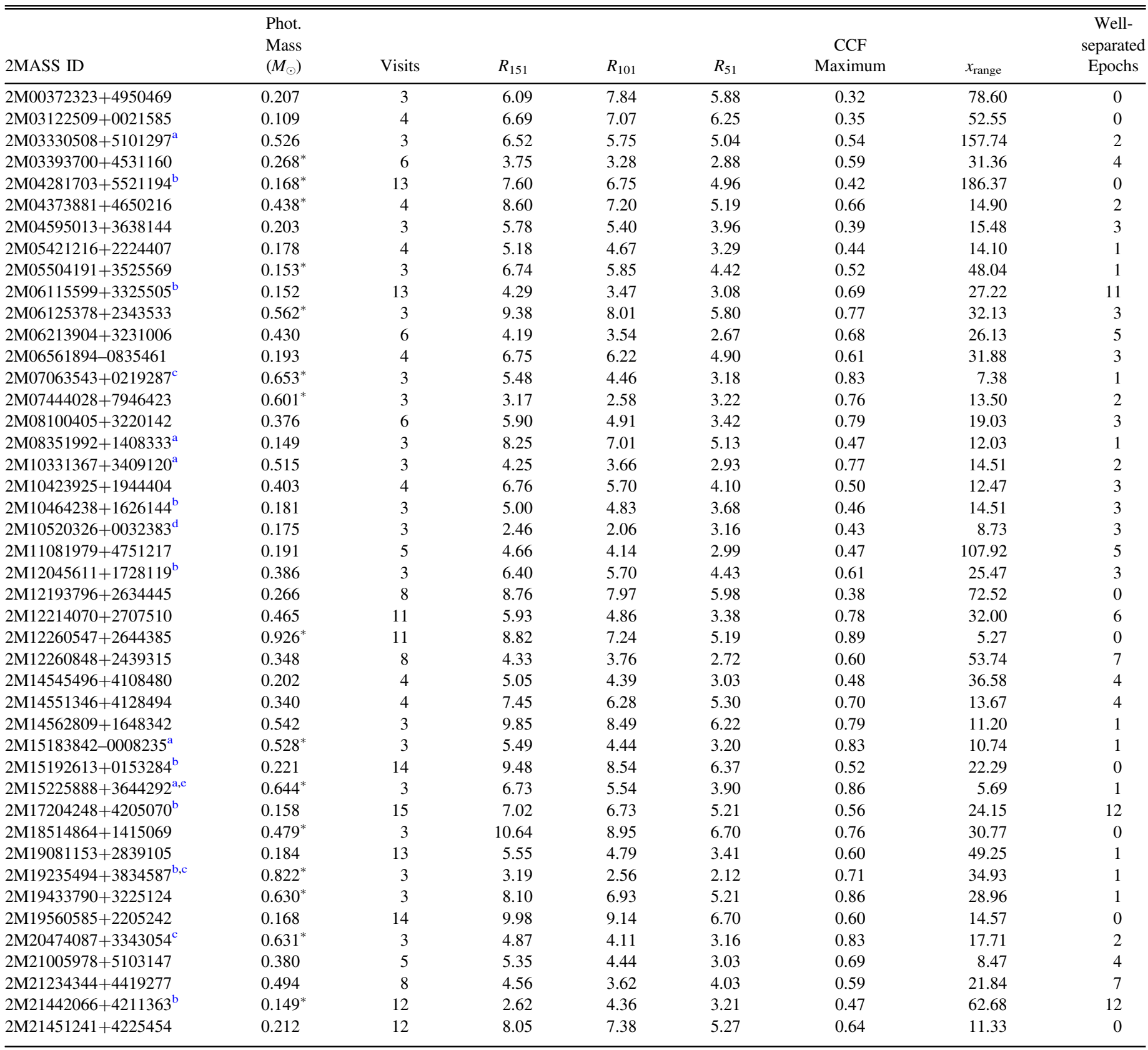

Notes. Stellar masses are estimated from the $(V-K)$-versus-mass relation derived by Delfosse et al. (2000); values tagged with an asterisk are determined from the $M_{K}$-versus-mass relation derived by Mann et al. (2015) after adopting a distance based on a measured trigonometric parallax or a fiducial solar neighborhood distance of $20 \mathrm{pc}$.

${ }^{a}$ Found here to be an SB3.

${ }^{\mathrm{b}}$ Identified by Deshpande et al. (2013) as an SB2.

${ }^{\mathrm{c}}$ Identified by El-Badry et al. (2018a) as an SB2.

${ }^{\mathrm{d}}$ Found here to be an SB4.

e Identified by El-Badry et al. (2018a) as an SB3.

(This table is available in its entirety in machine-readable form.)

These criteria identify 44 candidate M-dwarf SB2s, or just more than $3 \%$ of all $1350 \mathrm{M}$-dwarf ancillary targets in the DR13 catalog. These targets are listed in Table 1. Eight of these targets are among the nine SB2s flagged by Deshpande et al. (2013) in their analysis of a subset of this sample, indicating that our methods are capable of recovering the majority of the short-period, high flux ratio SB2s in the APOGEE database. The exception is $2 \mathrm{MJ} 19333940+3931372$, for which the APOGEE CCFs show evidence for profile changes, but the secondary component does not cleanly separate from the primary peak in any of the three visits obtained by APOGEE. Modifying our selection criteria to capture this source as a 
candidate SB2 would significantly increase the number of false positives that would need to be removed from our sample via visual inspection, so we choose to retain our more conservative cuts that will produce a smaller but higher-fidelity sample of candidate SB2s.

\subsection{Photometric Mass Estimates for Primary Stars}

We estimate the mass of the primary of each system in our sample using photometry and photometric calibrations from the literature. Photometric mass estimates are valuable for multiple reasons: the presence of multiple components in the system's spectra renders the standard APOGEE/ASPCAP analysis unreliable, and the DR13 APOGEE parameters have been shown to be unreliable for even single $M$ dwarfs (see Souto et al. 2017). For this photometric analysis, we adopted magnitudes from catalogs such as NOMAD (Zacharias et al. 2005), APOP (Qi et al. 2015), UCAC4 (Zacharias et al. 2013), UCAC5 (Zacharias et al. 2017), Viaux et al. (2013), and Lépine \& Shara (2005). We did not attempt to infer or correct for stellar reddening in this process, as any extinction is expected to be minimal due to the stars' presence within the solar neighborhood.

Stellar masses were derived using the $\left(V-K_{s}\right)$ versus mass color calibration derived by Delfosse et al. (2000). For stars without a reliable $V$ magnitude reported in the literature, we adopted the $M_{K}$ absolute magnitude-versus-mass calibration derived by Mann et al. (2015). The absolute magnitudes were derived using distances in the literature. For the stars without distances reported, we adopted $d=20.0 \mathrm{pc}$. The precision in the Delfosse et al. (2000) calibration is about 10\%, which returns an uncertainty of $\sim \pm 0.05 M\left(M_{\odot}\right)$.

\subsection{Additional RV Monitoring with HET/HRS}

We supplemented the APOGEE observations for a few systems with visible light spectroscopy from the fiber-fed High Resolution Spectrograph (HRS; Tull 1998) on the $9.2 \mathrm{~m}$ Hobby-Eberly Telescope (HET; Ramsey et al. 1998). We used the HRS with the $316 \mathrm{~g} 5936$ cross-disperser in the $30 \mathrm{~K}$ resolution mode with the $2^{\prime \prime}$ slit and central grating angle. This produced spectra spanning the wavelength range from 4076 to $7838 \mathrm{~nm}$, although we only used the region from $\sim 6600 \mathrm{~nm}$ redward because these M-dwarf spectra suffer from low signalto-noise at shorter wavelengths. All observations were conducted in queue mode (Shetrone et al. 2007). We exposed for 10-20 minutes per target per epoch based on magnitude. Wavelength calibration was obtained from ThAr frames that bracket the observation.

Spectra were extracted using a custom optimal extraction pipeline, modeled after the SpeXTool pipeline developed by Cushing et al. (2004) and similarly written in the interactive data language. The HRS pipeline automates basic imageprocessing procedures, such as overscan correction, bias subtraction, flat-fielding, and core spectral extraction processes such as tracing each order, computing the optimal fiber profile, and extracting source and ThAr lamp spectra. Wavelength solutions are derived by fitting a multi-order function to the ThAr spectra using the line list reported by Murphy et al. (2007) and applied to the object spectra.

Extracted, wavelength-calibrated spectra were then merged across areas of interorder interlap and trimmed to exclude regions of significant contamination by telluric absorption or
Table 2

Excluded Targets

\begin{tabular}{lc}
\hline \hline 2MASS ID & Max $\Delta \mathrm{RV} \mathrm{km} \mathrm{s}^{-1}$ \\
\hline 2M00372323+4950469 & 22.58 \\
2M03122509+0021585 & 15.13 \\
2M04281703+5521194 & 30.77 \\
2M12193796+2634445 & 30.05 \\
2M15192613+0153284 & 28.48 \\
2M18514864+1415069 & 24.97 \\
2M19560585+2205242 & 40.27 \\
2M21451241+4225454 & 15.04 \\
\hline
\end{tabular}

$\mathrm{OH}$ night-sky emission lines. Regions dominated by telluric absorption were identified by inspecting the LBLRTM atmospheric model (Clough et al. 2005); sharp night-sky emission features were removed by linearly interpolating over wavelength regions known to host strong emission lines (e.g., Abrams et al. 1994).

\section{Bulk Analysis}

\subsection{Cuts}

Of the 44 sources that we identified as likely SB2s, nine systems do not exhibit, at any epoch for which we have data, a velocity separation sufficiently large to reliably measure the $\mathrm{RVs}$ of both components with our initial RV extraction method. Analysis with TODCOR allowed us to recover RVs for one of these nine systems, providing a sample of 36 multiples with RVs for further analysis. Seven of these 36 systems are higher-order systems (six triples and one quadruple) with moderate velocity separations but poorly determined RVs due to significant blending in one or more of the APOGEE observations. TODCOR analysis allowed us to recover RVs for five of the six triples; the quadruple system remains unsolved. Exclusion of the eight poorly separated systems (see Table 2) and the two unsolved higher-order multiples ${ }^{25}$ leaves 34 targets for which we are able to measure mass ratios.

\subsection{RV Extraction from APOGEE Visits}

Using the procedures developed by F17, RVs were extracted from APOGEE CCFs for all components of each system. We describe the process briefly here but refer the reader to the earlier work for a detailed description. Using a multistep fitting process, RVs were extracted from each APOGEE visit CCF after converting the CCF's abscissa from lag space to velocity space.

In the first step, a Lorentzian was fit to the maximum peak of the CCF. This Lorentzian was then subtracted from the CCF, removing the primary peak. With the primary peak removed, a second Lorentzian was then fit to the maximum in the residual $\mathrm{CCF}$, which was implicitly identified as the secondary peak. For sources with multiple APOGEE visit spectra, the epoch containing the greatest separation between the primary and secondary peaks was identified as the "widest separated CCF." A dual-Lorentzian model was then fit to the widest separated CCF using the peak centers identified earlier for the primary

\footnotetext{
25 M10331367+3409120, 2M10520326+0032383.
} 

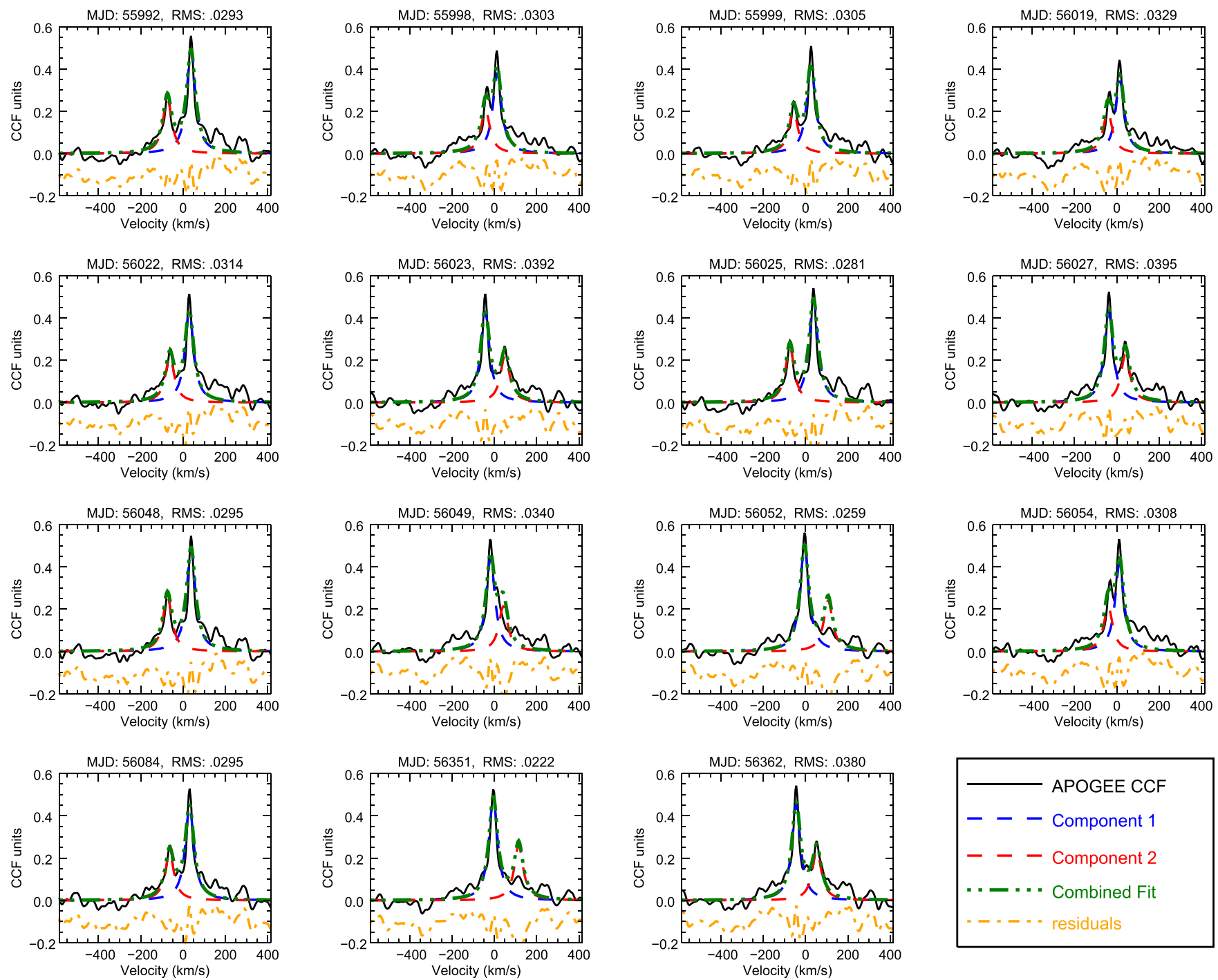

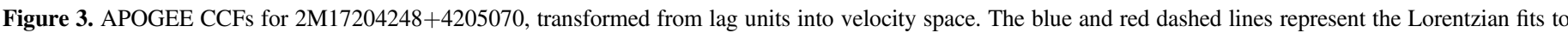

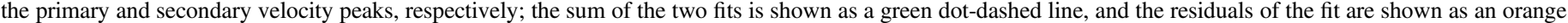

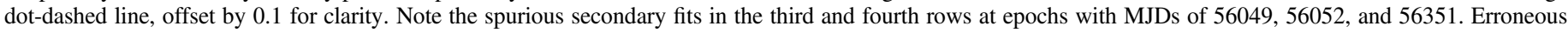
velocity values associated with spurious fits such as these have been removed from our data set by visual inspection.

and secondary components to initialize the fit. Finally, the dualLorentzian fit was performed on the remaining epochs using the peak heights and widths measured from the "widest separated epoch" to initialize the fit, along with the previously identified peak velocities.

A notable deficiency of this extraction method is that the resultant RVs lack an individually defined uncertainty value. Section 3.3 of F17 details their calculation of a pseudo-normal $1 \sigma$ error of $\sim 1.8 \frac{\mathrm{km}}{\mathrm{s}}$. We adopt this ensemble uncertainty value for all RVs extracted by CCF fitting. Another difficulty the CCF fitting method faces is consistent assignment of velocities to the primary and secondary components for SB2s with flux ratios close to unity. The accuracy of the RV values measured via this extraction technique suffered for epochs with small velocity separations, so we flagged these systems for follow-up analysis with the TODCOR algorithm (Zucker \& Mazeh 1994), which is more adept at extracting velocities from epochs with small velocity separations. The CCF fit-derived RVs are replaced at any epochs for which TODCOR RVs were extracted.
Figure 3 shows the Lorentzian fits to the primary and secondary peaks in all CCFs computed from APOGEE spectra of $2 \mathrm{M} 17204248+4205070$. Figures such as this were visually inspected to identify cases where the fits to the CCF peaks were obviously incorrect (i.e., a fit to a spurious structure in the CCF, most often occurring at epochs without well-separated $\mathrm{CCF}$ peaks). In such cases, spurious RV measures were removed from the sample. The SB2 RVs are listed in Table 3, which is presented here as a stub.

\section{2. $R V$ Extraction via TODCOR}

We used the TODCOR algorithm (Zucker \& Mazeh 1994) to measure RVs from all HET/HRS spectra and any APOGEE spectra flagged with low-RV separations. This TODCOR analysis followed the procedures previously discussed by Bender et al. (2005) and used the algorithm implementation of Bender et al. (2012); we briefly summarize here the key parts of this implementation and its modification for use with APOGEE spectra but refer the reader to the previous presentations for more details. TODCOR simultaneously cross-correlates each 
Table 3

RV Measurements of SB2s

\begin{tabular}{|c|c|c|c|c|c|c|}
\hline 2MASS ID & Visit & Epoch (MJD) & SDSS Plate and Fiber & $\mathrm{S} / \mathrm{N}$ & $v_{\text {prim }} \mathrm{km} \mathrm{s}^{-1}$ & $v_{\mathrm{sec}} \mathrm{km} \mathrm{s}^{-1}$ \\
\hline | & 2 & 56200.2983 & $6244-56200-131$ & 210 & -20.4 & 38.9 \\
\hline | & 4 & 56196.3190 & $6245-56196-077$ & 168 & 52.0 & -36.3 \\
\hline | & 5 & 56202.2755 & $6245-56202-074$ & 137 & 7.5 & $\cdots$ \\
\hline | & 6 & 56224.3188 & $6245-56224-077$ & 186 & 11.1 & 3.9 \\
\hline | & 3 & 56254.2442 & $6212-56254-050$ & 62 & -26.1 & -63.8 \\
\hline | & 4 & 56260.2176 & $6212-56260-050$ & 44 & -26.6 & -61.6 \\
\hline : & $:$ & : & $:$ & : & : & : \\
\hline
\end{tabular}

Note. Dashed out velocities indicate spurious RVs omitted from analysis. The RVs not extracted via TODCOR are assigned the ensemble uncertainty of $\sim 1.8 \mathrm{~km} \mathrm{~s}^{-1}$. (This table is available in its entirety in machine-readable form.)

Table 4

RV Measurements of SB3s

\begin{tabular}{|c|c|c|c|c|c|c|c|c|c|c|}
\hline 2MASS ID & Visit & Epoch (MJD) & SDSS Plate and Fiber & $\mathrm{S} / \mathrm{N}$ & $v_{\text {prim }} \mathrm{km} \mathrm{s}^{-1}$ & $\sigma_{\text {prim }}$ & $v_{\mathrm{sec}} \mathrm{km} \mathrm{s}^{-1}$ & $\sigma_{\mathrm{sec}}$ & $v_{\text {ter }} \mathrm{km} \mathrm{s}^{-1}$ & $\sigma_{\text {ter }}$ \\
\hline \multirow{3}{*}{$2 \mathrm{M} 03330508+5101297$} & 1 & 56257.1757 & $6538-56257-087$ & 42 & 30.86 & 0.53 & -84.72 & 1.57 & -11.49 & 1.02 \\
\hline & 2 & 56261.1912 & 6538-56261-088 & 73 & -65.39 & 0.30 & 78.53 & 0.99 & -10.98 & 0.73 \\
\hline & 3 & 56288.1033 & $6538-56288-069$ & 39 & -14.83 & 0.47 & -4.44 & 1.10 & -10.48 & 0.93 \\
\hline \multirow{2}{*}{$2 \mathrm{M} 04595013+3638144$} & 2 & 56262.2454 & $6542-56262-187$ & 44 & -33.77 & 0.46 & 39.26 & 1.43 & -10.74 & 0.59 \\
\hline & 3 & 56288.1841 & 6542-56288-192 & 52 & -33.04 & 0.44 & 38.62 & 1.47 & -8.80 & 0.71 \\
\hline $2 \mathrm{M} 08351992+1408333$ & 1 & 56284.3821 & $6612-56284-106$ & 248 & 37.04 & 0.36 & -17.11 & 0.84 & 17.30 & 0.48 \\
\hline \multirow[t]{3}{*}{ 2M15183842-0008235 } & 1 & 56080.2737 & 5906-56080-244 & 235 & 6.30 & 0.27 & -86.25 & 0.63 & -36.58 & 0.30 \\
\hline & 2 & 56435.2488 & 5906-56435-244 & 241 & -46.42 & 0.19 & -18.39 & 0.42 & -37.26 & 0.24 \\
\hline & 3 & 56467.1400 & 5906-56467-256 & 248 & -41.37 & 0.28 & -23.34 & 0.50 & -35.66 & 0.26 \\
\hline \multirow[t]{3}{*}{$2 \mathrm{M} 15225888+3644292$} & 1 & 56735.3805 & 5756-56735-106 & 152 & -68.10 & 0.43 & -19.08 & 2.10 & -56.64 & 0.74 \\
\hline & 2 & 56740.4314 & 5756-56740-009 & 185 & -82.70 & 0.24 & 8.13 & 2.50 & -57.52 & 0.50 \\
\hline & 3 & 56762.3135 & $5756-56762-010$ & 157 & -15.30 & 0.30 & -136.30 & 1.70 & -55.46 & 0.60 \\
\hline
\end{tabular}

Note. All velocities in this table were extracted via TODCOR.

(This table is available in its entirety in machine-readable form.)

target spectrum against the spectra of two template stars. For both the HRS and APOGEE observations, we generated template spectra from the BT-Settl library (Allard et al. 2012), convolved to each spectrograph's resolution and rotationally broadened using the Claret (2000) nonlinear limb-darkening models. Templates were optimized for each binary by maximizing the peak correlation using a template grid with $\Delta T_{\text {eff }}=100 \mathrm{~K}, \Delta \log (g)=0.5$, and $\Delta[M / H]=$ 0.5. This optimization happens independently for the HRS and APOGEE spectra. Due to variations in the quality of the line lists that underlie the BT-Settl models, we frequently derive slightly different optimal template sets for visible and nearinfrared spectra. These differences are typically within one or two grid points (i.e., $100-200 \mathrm{~K}$ in temperature and $<0.5 \mathrm{dex}$ in $\log (g)$ and $[M / H])$, and RVs are not sensitive to template choice at this resolution. An initial set of RVs was derived for all epochs of a given system by using the secondary-to-primary flux ratio $(\alpha)$ optimization of TODCOR. These RVs were then iterated with a fixed $\alpha$ corresponding to the average $\alpha$ derived for all epochs. We extract component RVs from a quadratic fit to the top six to eight points of the $2 \mathrm{D}$ cross-correlation peak and derive uncertainties using the maximum-likelihood formalism of Zucker \& Mazeh (1994). We flag the epochs for which velocities were extracted from HET/HRS or APOGEE spectra using this technique.

Our analysis also revealed several triple systems composed of a short-period binary along with a wider companion. We analyzed these systems with the TRICOR extension of TODCOR (Zucker et al. 1995) using three templates and solving for two independent flux ratios. The small number of epochs and relatively small temporal baselines limit what we can conclude about the stability of these systems or the orbital period of the wide component. We present the RVs derived for these candidate SB3 systems in Table 4; in all cases, the RVs derived for the wide companion are consistent within the uncertainties with the systemic RV of the inner pair, as would be expected for bound systems. Additionally, we did not detect any significant RV motion from any of the apparent wide companions. Consequently, we conclude that these are likely to be bound, hierarchical triple systems. 


\subsection{Mass-ratio Measurements}

\subsubsection{Measurement Technique}

We used the method presented in Wilson (1941) to measure the mass ratios ( $\left.q \equiv \frac{M_{\mathrm{sec}}}{M_{\mathrm{prim}}}\right)$ of our targets via a linear regression of primary velocity as a function of secondary velocity. Mass ratios greater than one were measured for four targets in the sample of 34 with reliably extracted RVs; we interpret these high $q$ values as a sign of a primary/secondary assignment mismatch and have therefore swapped the assignment for these targets and included the resulting lower $q$ values in the remainder of our analysis. The mass ratios measured for the 34member sample are listed in Table 5, with Wilson plots for each system presented in Figure 4 and the mass-ratio distribution of the entire sample shown in Figure 5.

\subsection{Detection Limits}

A detailed analysis of the selection effects introduced by the cadence of APOGEE observations and the sensitivity of our binary detection method to systems with varying mass ratios, inclinations, and separations is beyond the scope of this paper. To provide first-order indicators of the biases and limits that affect the makeup of our sample, however, we calculate fiducial detection limits imposed by the requirement that we detect multiple components, well separated in velocity space, in at least one APOGEE spectrum. It is worth noting that the ancillary science program is composed of targets of opportunity and was not designed to be complete in distance or magnitude.

We first consider the $H$-band flux ratio that a system must satisfy to be detectable as a double-lined SB2. Given the characteristic scale of the substructure in a typical APOGEE $\mathrm{CCF}$ and the typical ratios of the integrated areas of the primary and secondary CCF peaks for systems identified by our detection routine, we estimate that our detection method will begin to become significantly incomplete for systems with a secondary-to-primary $H$-band flux ratio $\leqslant 0.2$, or a magnitude difference of $\Delta H \leqslant 1.2$. Consulting the $H$-band magnitudes and fluxes tabulated by Kraus \& Hillenbrand (2007), we find that binaries with M-dwarf primaries typically satisfy this $H$-band flux ratio limit if they possess a mass ratio $\geqslant 0.5$. To illustrate the nature of this detection limit, we show in Figure 6 the CCF of a system with a mass ratio near, but just above, this fiducial $q=0.5$ limit.

We next consider the limits on system separation and orbital period imposed by the requirement that we detect two clearly separated CCF peaks. Given the resolution of the APOGEE spectrograph, a system's CCF peaks are clearly separated for primary-secondary velocity separations of $\Delta R V_{\text {lim }} \sim$ $30 \mathrm{~km} \mathrm{~s}^{-1}$ or more (F17). This velocity separation threshold imposes a joint constraint on the inclination $(i)$, total mass $\left(M_{1}+M_{2}\right)$, and orbital period $(P)$ of systems in our sample:

$$
P \leqslant \frac{2 \pi G\left(M_{1}+M_{2}\right) \sin ^{3} i}{\Delta \mathrm{RV}_{\mathrm{lim}}^{3}}
$$

Expressing this constraint in terms of the system's semimajor axis $(a)$, rather than its orbital period, makes this limit

$$
a \leqslant \frac{G\left(M_{1}+M_{2}\right) \sin ^{3} i}{\Delta \mathrm{RV}_{\lim }^{2}} .
$$

Table 5

Mass Ratio and $\Delta \mathrm{RV}$ of Analyzed Stars

\begin{tabular}{lccr}
\hline \hline 2MASS ID & $q \pm \delta q$ & $\frac{\delta q}{q}$ & Max $\Delta \mathrm{RV}$ \\
\hline 2M03330508+5101297 & $0.593 \pm 0.012$ & 0.020 & 143.92 \\
2M03393700+4531160 & $0.976 \pm 0.018$ & 0.018 & 91.13 \\
2M04373881+4650216 & $0.786 \pm 0.043$ & 0.054 & 37.69 \\
2M04595013+3638144 & $0.720 \pm 0.043$ & 0.060 & 73.03 \\
2M05421216+2224407 & $0.979 \pm 0.059$ & 0.060 & 41.65 \\
2M05504191+3525569 & $0.671 \pm 0.162$ & 0.241 & 37.27 \\
2M06115599+3325505 & $0.840 \pm 0.006$ & 0.008 & 70.40 \\
2M06125378+2343533 & $0.796 \pm 0.022$ & 0.028 & 42.88 \\
2M06213904+3231006 & $0.890 \pm 0.050$ & 0.044 & 86.97 \\
2M06561894-0835461 & $0.617 \pm \cdots$ & $\ldots$ & 82.85 \\
2M07063543+0219287 & $0.887 \pm 0.283$ & 0.251 & 57.98 \\
2M07444028+7946423 & $0.906 \pm 0.047$ & 0.042 & 91.12 \\
2M08100405+3220142 & $0.982 \pm 0.013$ & 0.013 & 34.01 \\
2M08351992+1408333 & $0.730 \pm 0.017$ & 0.024 & 54.15 \\
2M10423925+1944404 & $0.774 \pm 0.142$ & 0.184 & 29.56 \\
2M10464238+1626144 & $0.840 \pm 0.024$ & 0.028 & 68.10 \\
2M11081979+4751217 & $0.217 \pm 0.014$ & 0.066 & 64.32 \\
2M12045611+1728119 & $0.654 \pm 0.054$ & 0.082 & 141.45 \\
2M12214070+2707510 & $0.980 \pm 0.009$ & 0.009 & 42.65 \\
2M12260547+2644385 & $0.762 \pm 0.012$ & 0.016 & 40.54 \\
2M12260848+2439315 & $0.998 \pm 0.045$ & 0.045 & 41.42 \\
2M14545496+4108480 & $0.889 \pm 0.006$ & 0.007 & 99.39 \\
2M14551346+4128494 & $0.743 \pm 0.017$ & 0.023 & 99.42 \\
2M14562809+1648342 & $0.565 \pm 0.389$ & 0.688 & 53.92 \\
2M15183842-0008235 & $0.772 \pm 0.019$ & 0.025 & 92.55 \\
2M15225888+3644292 & $0.461 \pm 0.012$ & 0.025 & 121.00 \\
2M17204248+4205070 & $0.660 \pm 0.001$ & 0.002 & 110.28 \\
2M19081153+2839105 & $0.887 \pm 0.019$ & 0.022 & 47.71 \\
2M19235494+3834587 & $0.998 \pm 0.016$ & 0.016 & 161.26 \\
2M19433790+3225124 & $0.831 \pm \cdots$ & $\ldots$ & 58.67 \\
2M20474087+3343054 & $0.909 \pm 0.037$ & 0.041 & 76.92 \\
2M21005978+5103147 & $0.988 \pm 0.017$ & 0.017 & 57.22 \\
2M21234344+4419277 & $0.905 \pm 0.016$ & 0.018 & 124.27 \\
2M21442066+4211363 & $0.947 \pm 0.037$ & 0.039 & 124.23 \\
\hline & & & \\
\hline
\end{tabular}

Notes.

${ }^{\text {a }}$ For these targets, $q>1$. We assume this is due to a primary/secondary mismatch and report $q^{-1}$ as $q$.

${ }^{\mathrm{b}}$ Only two epochs were usable for these targets; therefore, $\delta q$ is not well defined.

(This table is available in its entirety in machine-readable form.)

Edge-on $\left(i \sim 90^{\circ}\right)$, equal-mass systems are the most favorable configuration for detection: for a fiducial pair of $0.5 M_{\odot}$ stars, we find a limiting period of $\sim 1 \mathrm{yr}$ and a limiting separation of $1 \mathrm{au}$; for a lower-mass pair of $0.25 M_{\odot}$ stars, we find a limiting period and separation of $\sim 0.5 \mathrm{yr}$ and au, respectively. ${ }^{26}$ Due to the $\sin ^{3} i$ term in these limits, however, these limits decrease quickly with inclination: a modest inclination of $30^{\circ}$ reduces the detection limits for the $0.5 M_{\odot}$ binary to $\sim 0.12 \mathrm{yr}$ and au and to $0.06 \mathrm{yr}$ and au for the $0.25 M_{\odot}$ system.

The considerations above demonstrate that our sample is biased toward edge-on systems with mass ratios $\geqslant 0.5$ and will be most complete for systems with characteristic periods and separations of $\leqslant 0.1 \mathrm{yr} \mathrm{au}^{-1}$. We therefore adopt $0.1 \mathrm{yr} \mathrm{au}^{-1}$ as useful benchmarks for the completeness limits of our observed sample and for comparing the properties of this sample to those measured from other samples of binary stars reported in the literature.

\footnotetext{
${ }^{26}$ As years and astronomical units are defined based on the properties of our own solar system and scale identically with system mass and inclination, the limiting period and separation for a fiducial system will be numerically identical when expressed in units of years and astronomical units.
} 


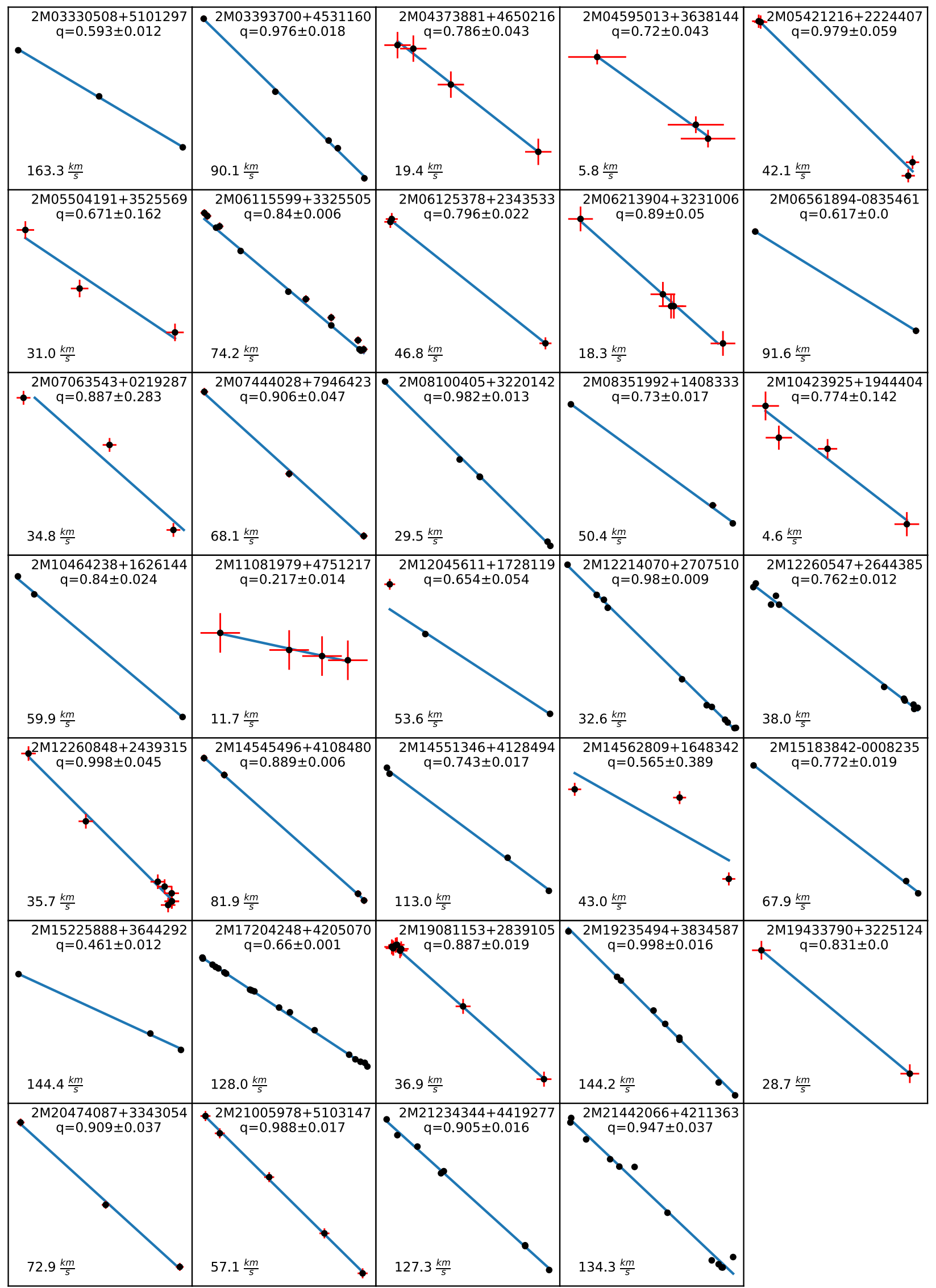

Figure 4. Wilson plots of each mass-ratio measurement in our sample. The black circles are RV observations. Uncertainties are shown as red bars. The blue line of each plot is the best-fit line to the data, from which the mass ratio is calculated. The horizontal axis is $v_{\text {sec }}$, and the vertical axis is $v_{\text {prim. }}$. The aspect ratio between the vertical and horizontal axes of each subplot is 1 . In the lower left corner of each subplot, the $v_{\text {sec }}$ range is given. 


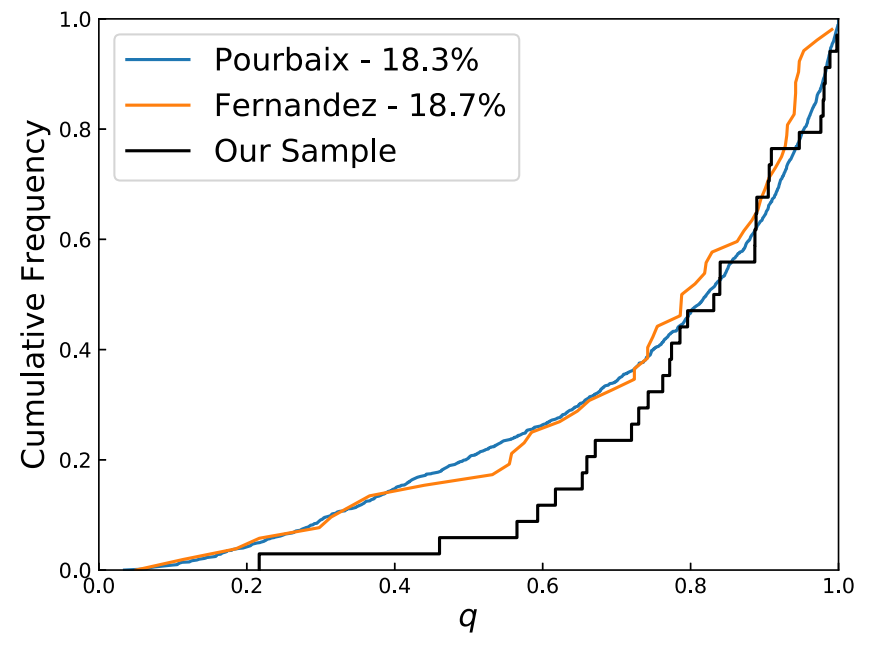

Figure 5. Mass-ratio distribution of our sample and the Kolmogorov-Smirnov statistics comparing our distribution and those of Pourbaix et al. (2004) and F17.

\section{Full Orbit Fits for High-visit, High- $\Delta$ RV Systems}

\subsection{Criteria for Full Orbital Fits}

The choice of targets for orbital fits was made using the three following criteria, met by four systems.

1. Primary and secondary RVs for at least eight visits.

2. Fractional mass-ratio uncertainty less than $10 \%$.

3. A $V_{\text {cov }}$ value of at least 0.7 , where $V_{\text {cov }}$ is the velocity coverage statistic presented in Equation (5) of F17,

$$
V_{\mathrm{cov}}=\frac{N}{N-1}\left(1-\frac{1}{\mathrm{RV}_{\text {span }}^{2}} \sum_{i=1}^{N}\left(\mathrm{RV}_{i+1}-\mathrm{RV}_{i}\right)^{2}\right),
$$

with $N$ equal to the number of visits, and $\mathrm{RV}_{\text {span }}=\mathrm{RV}_{\max }-\mathrm{RV}_{\min }$.

\subsection{Fitting Procedure}

The RVs expected for each component were calculated from a model consisting of six parameters: velocity semi-amplitude of the primary $(K)$, eccentricity $(e)$, longitude of periastron $(\omega)$, time of periastron $(T)$, orbital period $(P)$, and barycenter velocity $(\gamma)$. A model RV curve was computed, starting with the mean anomaly $M$ :

$$
M=\frac{2 \pi}{P}(t-T)
$$

Using $M$, the eccentric anomaly $E$ was computed:

$$
E=M+e \sin (M)+e^{2} \frac{\sin (2 M)}{2} .
$$

Using $E$, the true anomaly $\nu$ was computed:

$$
\nu=2 \arctan \left(\sqrt{\frac{1+e}{1-e}} \cdot \tan \left(\frac{E}{2}\right)\right) .
$$

Finally, the primary and secondary RVs were calculated,

$$
\begin{gathered}
\operatorname{vel}_{\text {prim }}=K[\cos (\nu+\omega)+e \cos (\omega)]+\gamma, \\
\operatorname{vel}_{\text {sec }}=-\frac{K}{q}[\cos (\nu+\omega)+e \cos (\omega)]+\gamma,
\end{gathered}
$$

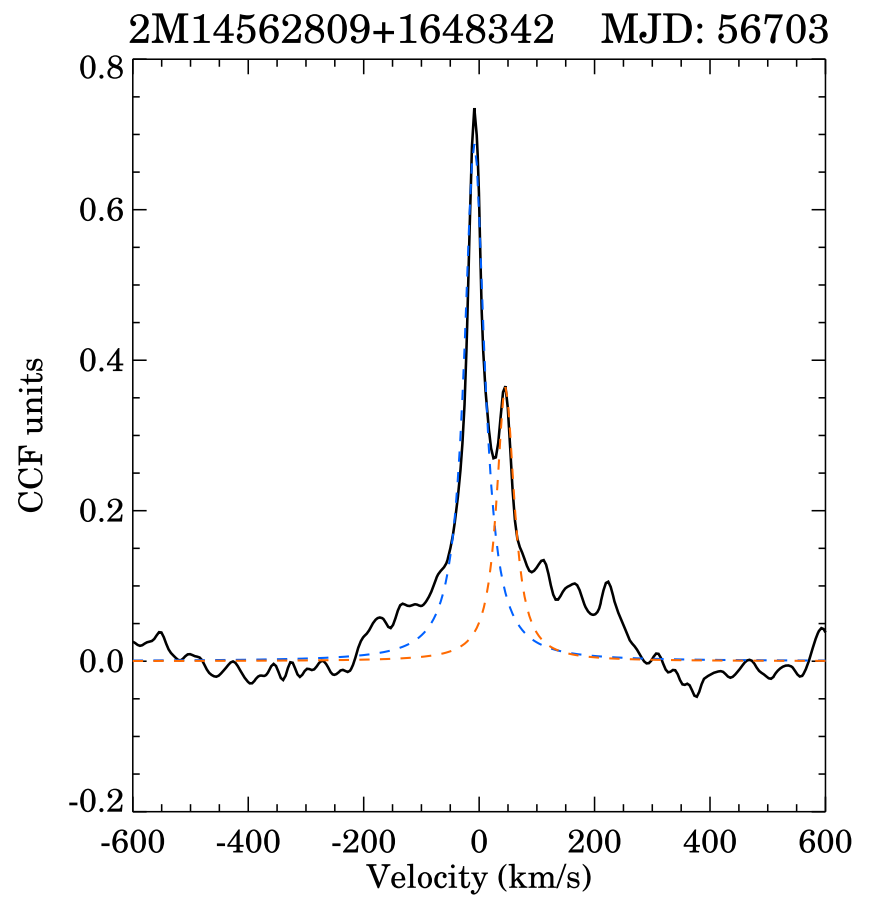

Figure 6. APOGEE CCF for $2 \mathrm{M} 14562809+1648342$, a binary whose mass ratio $(q=0.565)$ places it near the fiducial $q=0.5 / \Delta H=1.2$ limit that we estimate for where our search method will become substantially incomplete due solely to the inability to confidently detect the secondary companion, even at high-velocity separations.

where $q$ was treated as a constant for each system, its value inherited from the method presented in Section 3.2.

The set of orbital parameters $\boldsymbol{\theta} \equiv(K, e, \omega, T, P, \gamma)$ that accurately predicts the observed RVs of each component represents a possible orbital solution for the system. To find the best orbital fit, we explored this parameter space using Bayesian techniques. We sampled the parameter space using emcee (Foreman-Mackey et al. 2013), a Python implementation of an affine invariant ensemble sampler (Goodman \& Weare 2010). We used an ensemble of 4000 walkers, distributed evenly throughout the space, for 2000 steps. We kept the final 1000 steps of each run, discarding the initial 1000 as a burn-in phase.

For the number of visits typical of our orbital solutions (on the order of 10), the posterior probability distributions of $P$ were multimodal and highly degenerate. This made a period determination difficult. To perform the period search, we probed the parameter space using a modified likelihood function. The likelihood $p$ of observing the data set $\boldsymbol{y}$ given $\boldsymbol{\theta}$ was defined as

$$
p(\boldsymbol{y} \mid \boldsymbol{\theta})=\exp \left[-\sqrt{\frac{1}{N} \sum_{i=1}^{N} \frac{\left(o_{i}-c_{i}\right)^{2}}{\sigma_{i}} \operatorname{prim}+\frac{\left(o_{i}-c_{i}\right)^{2}}{\sigma_{i}} \sec }\right],
$$

where $o_{i}$ is the $i$ th observation in $\boldsymbol{y}$ and $c_{i}$ is the computed RV based on $\boldsymbol{\theta}$ at the $i$ th epoch. This definition prevents the ensemble from converging tightly on any single local maximum, allowing for multiple modes to be explored in a single walk. Figure 7 shows an example of the results of the MCMC period search using this likelihood definition, overlaid with a Lomb-Scargle periodogram. In Figure 7, the samples are densest in period space at 3.29 days, corresponding to a peak in 


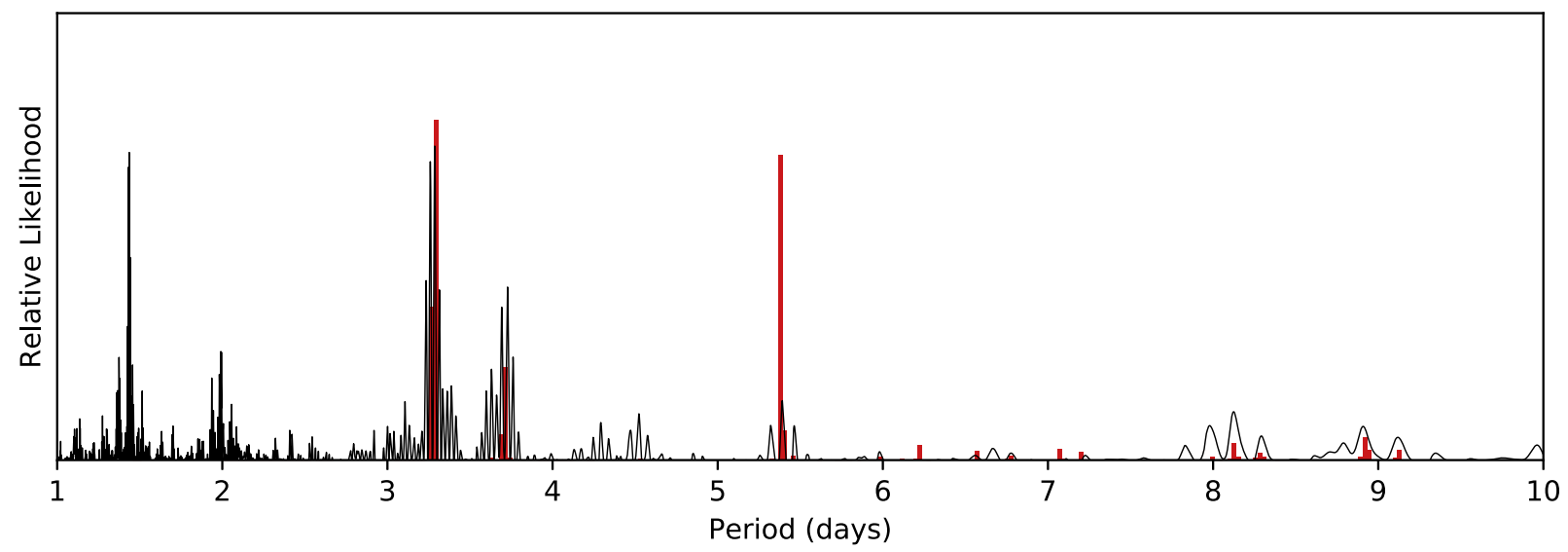

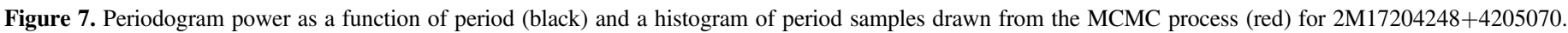

periodogram power. We define a period confidence, $\mathcal{L}$, as the fraction of MCMC samples contained within the primary peak identified by the period search: in the case shown in Figure 7, $\mathcal{L}=30 \%$. The highest period confidence value we measure is $79 \%$, for $2 \mathrm{M} 21442066+4211363$; for the other three systems, we measure period confidence values ranging from $16 \%$ to $56 \%$, suggesting that the maximum-likelihood period is probable but not yet definitively measured. The MCMC analysis also appears to favor shorter periods for these systems, producing a potential bias for other values inferred from the period measurements.

After constraining the period, we adopt the following priors for the other five parameters:
1. $0<K<100, \mathrm{~km} \mathrm{~s}^{-1}$
2. $0<e<0.8$,
3. $0<\omega<2 \pi$,
4. $\left(\right.$ median JD $\left.-\frac{P}{2}\right)<T<\left(\right.$ median $\mathrm{JD}+\frac{P}{2}$ ),
5. min. observed RV $<\gamma<\max$. observed RV.

In the random walk for the full orbital solution, we use the likelihood function:

$$
p(\boldsymbol{y} \mid \boldsymbol{\theta})=\exp \left[-\sum_{i=1}^{N} \frac{\left(o_{i}-c_{i}\right)^{2}}{2 \sigma_{i}} \operatorname{prim}+\frac{\left(o_{i}-c_{i}\right)^{2}}{2 \sigma_{i}} \sec \right] .
$$

This likelihood function reflects our assumption of independent Gaussian probabilities. For cases when the ensemble converged toward $e=0$, we performed a second run with a slightly modified circular orbit model, in which both $e$ and $\omega$ were constrained to 0 . For each orbit fit, our choice of model was consistent with the Bayesian information criterion.

We report the 50th quantile of the post burn-in distribution of the converged walkers as the value of each parameter. As uncertainties, we report the 16th and 84th quantiles as quasi$1 \sigma$ values.

As a check against our orbital solutions, we estimate a lower bound on primary mass in Table 6 (see the Appendix for the derivation). All dynamical lower mass limits are significantly lower (by a factor of 5-10) than the photometric mass estimate of the primary listed in Table 1. This indicates that either the sample includes an anomalously large number of highinclination systems, such that their dynamical mass is a significant underestimate of their true mass, or that the orbital period we have inferred is underestimated, as the dynamical mass estimate scales linearly with the system's assumed orbital period. We suspect the latter explanation is more likely and suggest that additional monitoring of these systems to remove the uncertainty that remains in the systems' periods is necessary.

\section{Results}

Frequency.-As noted earlier, the systems we detect as close multiples are a biased and incomplete subset of the larger, true population of close multiples within the parent sample of the SDSS-III/APOGEE M-dwarf ancillary sample. Nonetheless, it is instructive to compare the raw MF that we infer from this sample to prior measurements of the frequency of close companions to M-type primaries. FM1992 and CBK2012 analyzed RV variability in multi-epoch optical spectra to infer a close $(a<0.4 \mathrm{au})$ binary fraction of $1.8 \% \pm 1.8 \%$ and $3 \%-4 \%$, respectively. More recently, Shan et al. (2015) analyzed the population of $M+M$ eclipsing binaries in the Kepler field to infer a frequency of $11 \% \pm 2 \%$ for close $\left(P<90\right.$ days, or $a \leqslant 0.25$ au for a fiducial $0.5+0.5 M_{\odot}$ system) companions to M-dwarf primaries.

The raw (i.e., without corrections for incompleteness, inclination bias, etc.) binary fraction that we measure in our APOGEE sample $(\sim 3 \% ; 37 / 1350=2.74 \%)$ seems to match the frequencies inferred by FM1992 and CBK2012. Those prior measurements have been corrected for incompleteness and selection effects, however, while our raw MF has not. The only selection effect that would drive our raw MF to overestimate the true value is the magnitude-limited nature of the APOGEE M-dwarf ancillary targets; biases due to inclination, flux ratios, and temporal sampling will all conspire to make our empirically measured rate underestimate the true MF. Thus, our measurement should be an underestimate of the true intrinsic MF. If completeness correction shows that our current binary detection rate is above $50 \%$, then our result would support that of FM1992 and CBK2012. If our current binary detection rate is below $50 \%$, then our result would more strongly favor that of Shan et al. (2015). Detailed modeling will be necessary to draw strong conclusions and should be the subject of future work.

Mass-ratio distribution.-Measuring the mass ratios of 29 M-dwarf SB2s and five SB3s, we find a mass-ratio distribution 

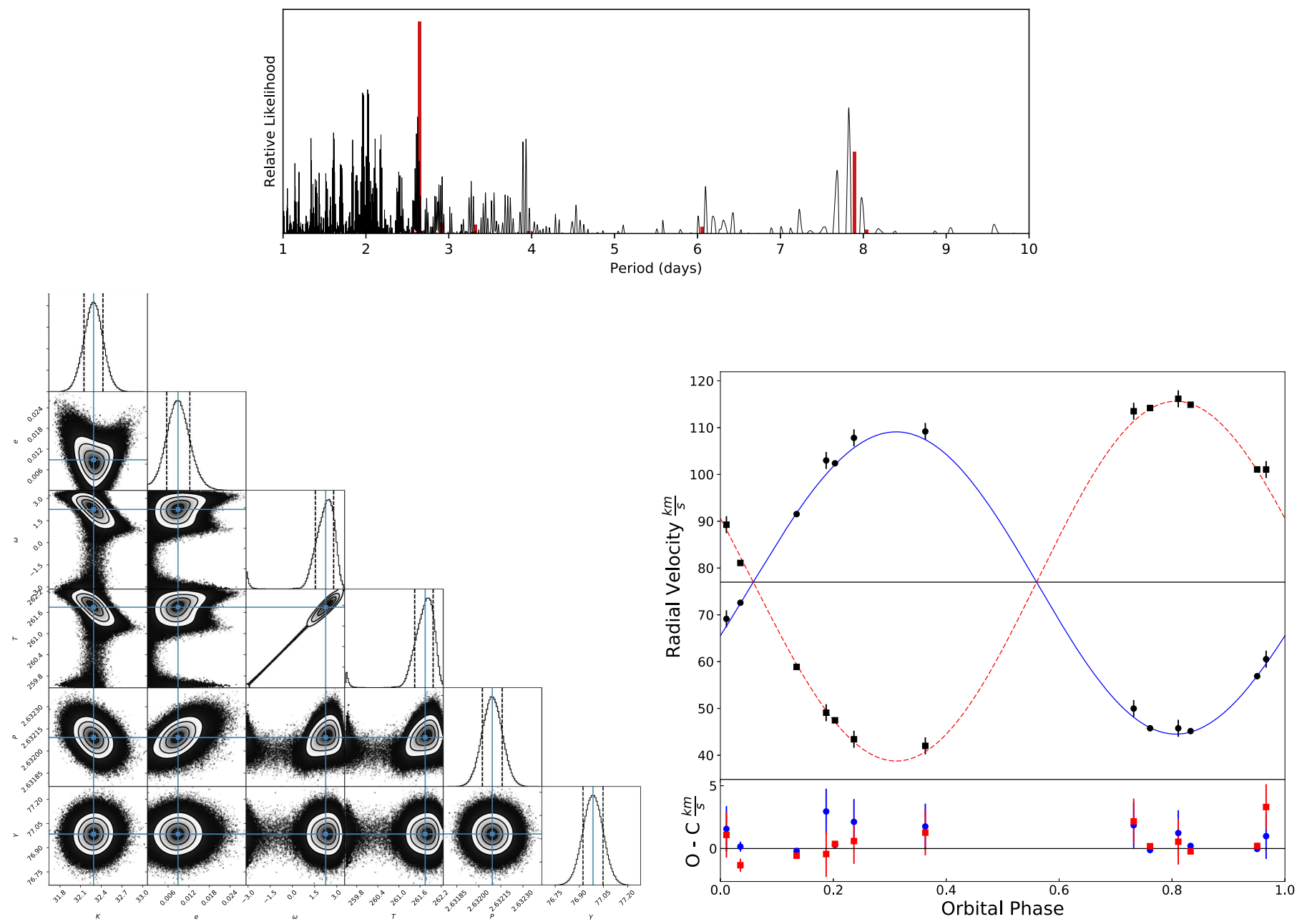

Figure 8. Figures for $2 \mathrm{M} 06115599+3325505$, with $\mathcal{L}=56 \%$ at $P=2.63$ days. (Top) Lomb-Scargle periodogram power as a function of period (black) and the histogram of MCMC samples obtained during the period search (red). (Bottom left) Corner plot of the posterior probability distribution given by the random walk. (Bottom right) RV plot of this system. The solid blue curve is the primary component velocity, and the red dashed curve is the secondary component velocity. Primary component velocities are marked as squares, and secondary velocities are marked as circles.

that reaches as low as 0.217 but with most systems having mass ratios between 0.8 and 1 . As noted in Section 3.4, requiring the detection of spectral counterparts for both components of the system will bias our sample toward equal mass ratios. Nonetheless, it is again instructive to compare our mass-ratio distribution to those measured in existing samples of SB2s, particularly as those catalogs will suffer from similar selection biases. Our cumulative mass-ratio distribution shows fair agreement with those found by Pourbaix et al. (2004) and F17: while our mass-ratio distribution appears somewhat more strongly skewed toward equal-mass systems, a KolmogorovSmirnov test finds an $\sim 18 \%$ chance that the mass-ratio distribution that we measure for the APOGEE M-dwarf SB2s is consistent with those measured by Pourbaix et al. (2004) and F17 for similarly biased catalogs of (higher-mass) SB2s.

Orbits.-The orbital fit results are tabulated in Table 6. All four orbital solutions exhibit small eccentricities $(e<0.1)$. We find periods of 2.6-8.2 days. In the context of a multimodal period distribution, defining uncertainty via peak width is problematic. As a result, we omit uncertainties on our measurement of orbital period in favor of the period confidence defined in Section 4.2. Three key figures (see Figures 8-11) are included for each orbital solution.

\section{Conclusions}

1. We have identified 44 candidate close multiple systems among the 1350 targets in the SDSS-III/APOGEE M-dwarf ancillary sample. These candidates include eight of the nine SB2s previously identified by Deshpande et al. (2013) in their analysis of a subset of the APOGEE M-dwarf sample, as well as three SB2s and an SB3 identified by El-Badry et al. (2018a) in their search for binaries within DR14, indicating that our algorithm successfully recovers close binaries whose APOGEE spectra capture an epoch where the system exhibits a large velocity separation.

2. We have extracted RVs for components in 34 of these systems, including five systems that appear to be higherorder multiples. In most cases, these RV measurements are obtained by fitting peaks in the CCFs produced by the APOGEE reduction and analysis pipeline; in systems with more than two components, or with velocity separations too small to resolve in the APOGEE CCF, we have extracted RVs using the TODCOR algorithm on the APOGEE spectra themselves. For two stars, we have also obtained follow-up spectroscopy with the HRS on 

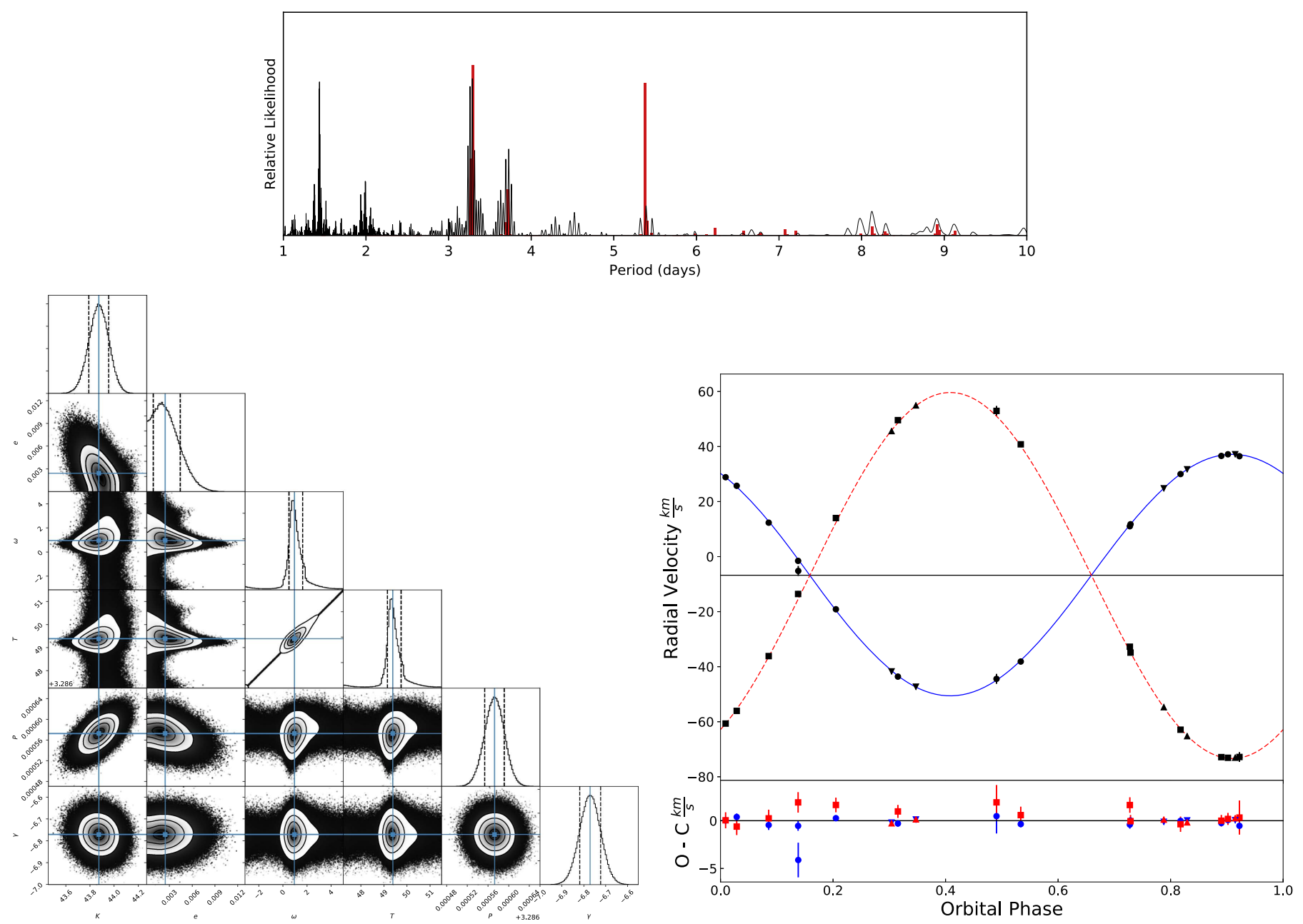

Figure 9. Figures for $2 \mathrm{M} 17204248+4205070$, with $\mathcal{L}=30 \%$ at $P=3.29$ days. (Top) Lomb-Scargle periodogram power as a function of period (black) and the histogram of MCMC samples obtained during the period search (red). (Bottom left) Corner plot of the posterior probability distribution given by the random walk. (Bottom right) RV plot of this system. The solid blue curve is the primary component velocity, and the red dashed curve is the secondary component velocity. Primary component velocities are marked as squares, and secondary velocities are marked as circles.

Table 6

Orbital Fits

\begin{tabular}{lcccc}
\hline \hline & 2M06115599+3325505 & 2M17204248+4205070 & 2M21234344+4419277 & 2M21442066+4211363 \\
\hline$K$ & $32.29 \pm 0.14$ & $43.87 \pm 0.08$ & $58.51 \pm 0.59$ & $61.16 \pm 0.46$ \\
$e$ & $0.01 \pm 0.003$ & $0.002 \pm 0.002$ & $0.062 \pm 0.0 .012$ & 0 \\
$\omega$ & $128.94_{-39.84}^{+30.75}$ & $54.47_{-25}^{+39.25}$ & $127.78_{-9.00}^{+8.69}$ & 0 \\
$T$ & $261.7467 \pm 0.006$ & $49.3840_{-0.2350}^{+0.3583}$ & $488.0751_{-0.2036}^{+0.1982}$ & $205.3381 \pm 0.0057$ \\
$P(\mathcal{L})$ & $2.63(56 \%)$ & $3.29(30 \%)$ & $8.17(16 \%)$ & $3.30(79 \%)$ \\
$\gamma$ & $76.98 \pm 0.06$ & $-6.77 \pm 0.05$ & $-123.59 \pm 0.45$ & $-17.22 \pm 0.37$ \\
$\frac{M_{\text {prima }}}{M_{\odot}}$ & 0.005 & 0.017 & 0.089 & 0.04 \\
\hline
\end{tabular}

Note. Units are $K\left(\mathrm{~km} \mathrm{~s}^{-1}\right), \omega(\mathrm{deg}), T(\mathrm{JD}-2,456,000), P($ days $), \gamma\left(\mathrm{km} \mathrm{s}^{-1}\right)$.

a Dynamical minimum mass estimates for the system's primary component, derived as explained in the Appendix. The minimum mass of an M-dwarf primary is $M \geqslant 0.075 M_{\odot}$; individual photometric mass estimates for each primary are listed in Table 1, with a range of $0.15-0.49 M_{\odot}$.

the HET; we analyze these optical/near-infrared data using the TODCOR routine as well.

3. We fit primary and secondary RVs to measure mass ratios for the closest pair of each of the 34 systems for which we extract RVs. The mass-ratio distribution of close pairs in our sample skews toward equal-mass systems and includes only one system with a mass ratio $<0.45$; this is consistent with first-order estimates of the bias toward higher mass ratios that should result from requiring a positive spectroscopic detection of both primary and secondary components. Nonetheless, the (biased and incomplete) mass-ratio distribution that we measure from 

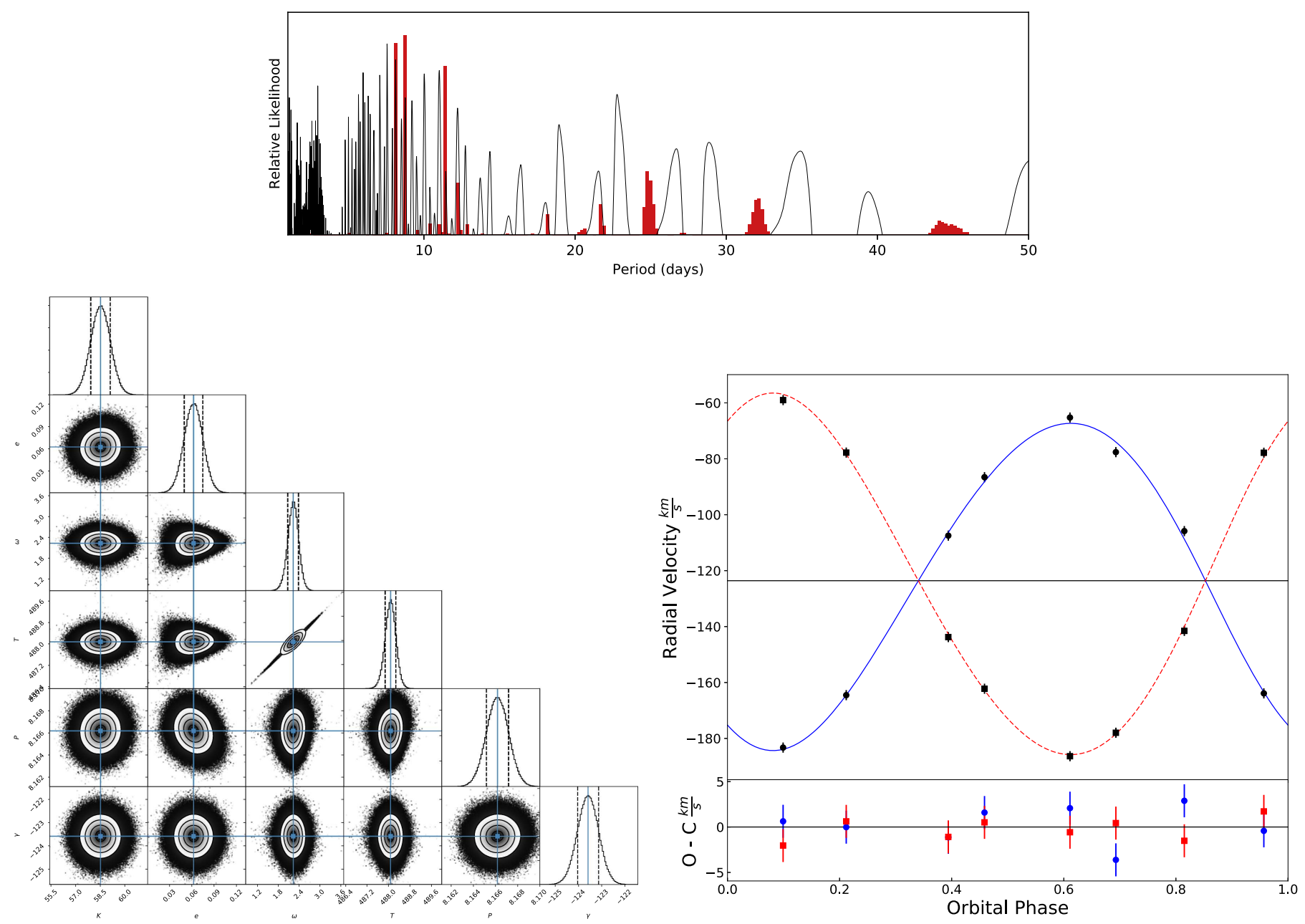

Figure 10. Figures for $2 \mathrm{M} 21234344+4419277$, with $\mathcal{L}=16 \%$ at $P=8.17$ days. (Top) Lomb-Scargle periodogram power as a function of period (black) and the histogram of MCMC samples obtained during the period search (red). (Left) Corner plot of the posterior probability distribution given by the random walk. (Bottom right) RV plot of this system. The solid blue curve is the primary component velocity, and the red dashed curve is the secondary component velocity. Primary component velocities are marked as squares, and secondary velocities are marked as circles.

the M-dwarf sample is consistent at the $1 \sigma$ level with the mass-ratio distributions reported in the literature for similarly biased samples of younger and more massive stars, suggesting that the mass ratios of close multiples are not a strong function of primary mass.

4. The low periods we measure for our targets $(P<$ 10 days) are consistent with the small separations we expect for M-dwarf SB2s. The low eccentricities we measure $(e<0.1)$ reflect the tidal interactions to which close binaries are subject. Our orbit fits exhibit small residuals, excluding third bodies down to very low masses.

J.S., K.R.C., and M.K. acknowledge support provided by the NSF through grant AST-1449476 and from the Research Corporation via a Time Domain Astrophysics Scialog award. C.F.B. and N.R. acknowledge support provided by the NSF through grant AST-1517592. N.D. acknowledges support for this work from the NSF through grant AST-1616684.

Funding for SDSS-III has been provided by the Alfred P. Sloan Foundation, the Participating Institutions, the National Science Foundation, and the U.S. Department of Energy Office of Science. The SDSS-III website is http://www.sdss3.org/.
SDSS-III is managed by the Astrophysical Research Consortium for the Participating Institutions of the SDSS-III Collaboration, including the University of Arizona, the Brazilian Participation Group, Brookhaven National Laboratory, Carnegie Mellon University, the University of Florida, the French Participation Group, the German Participation Group, Harvard University, the Instituto de Astrofisica de Canarias, the Michigan State/Notre Dame/JINA Participation Group, Johns Hopkins University, Lawrence Berkeley National Laboratory, the Max Planck Institute for Astrophysics, the Max Planck Institute for Extraterrestrial Physics, New Mexico State University, New York University, Ohio State University, Pennsylvania State University, the University of Portsmouth, Princeton University, the Spanish Participation Group, the University of Tokyo, the University of Utah, Vanderbilt University, the University of Virginia, the University of Washington, and Yale University.

DAGH was funded by Ramón y Cajal fellowship number RYC-2013-14182. DAGH and OZ acknowledge support provided by the Spanish Ministry of Economy and Competitiveness (MINECO) under grant AYA-2014-58082-P. 

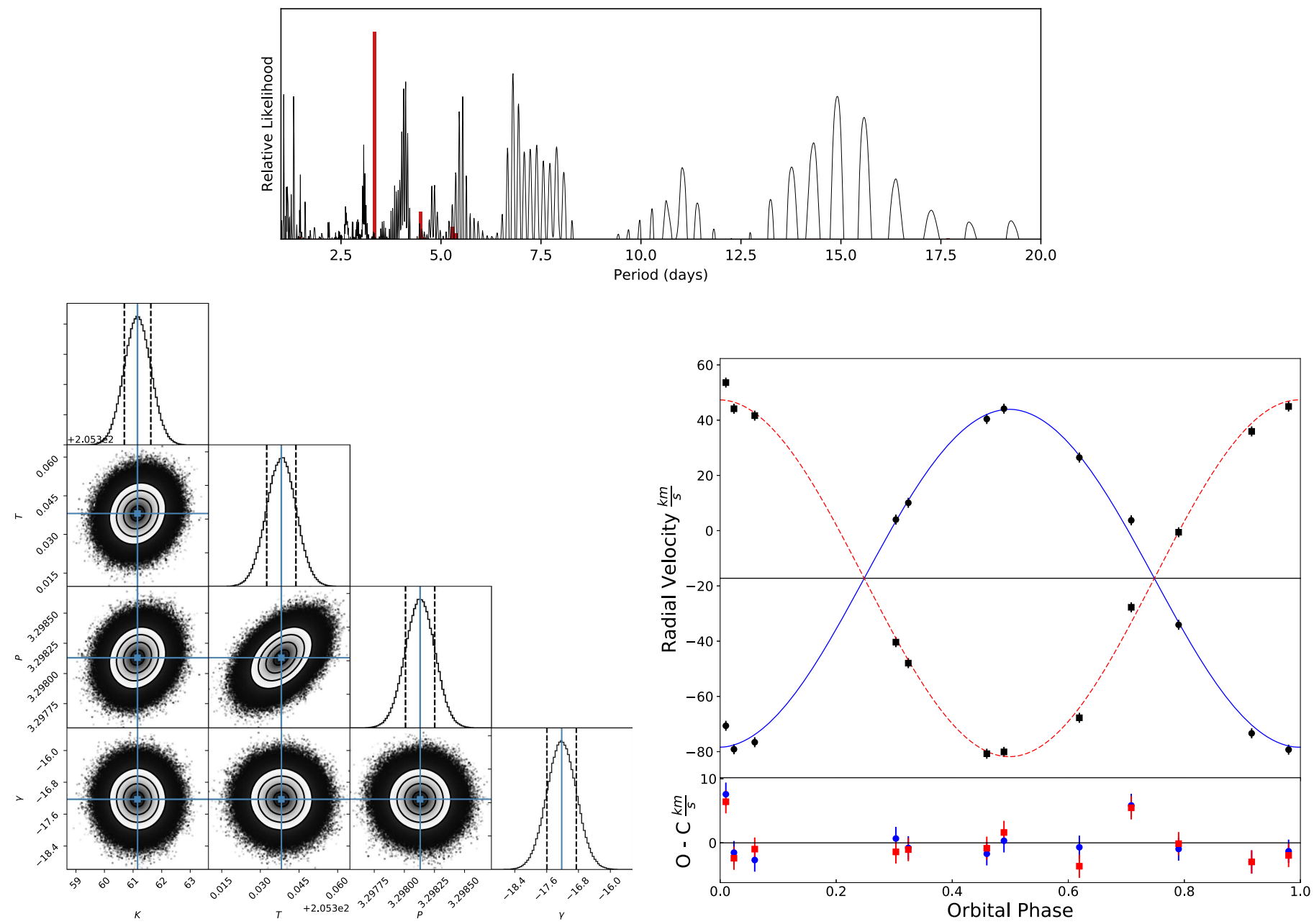

Figure 11. Figures for $2 \mathrm{M} 21442066+4211363$, with $\mathcal{L}=79 \%$ at $P=3.30$ days. (Top) Lomb-Scargle periodogram power as a function of period (black) and the histogram of MCMC samples obtained during the period search (red). (Left) Corner plot of the posterior probability distribution given by the random walk. (Bottom right) RV plot of this system. The solid blue curve is the primary component velocity, and the red dashed curve is the secondary component velocity. Primary component velocities are marked as squares, and secondary velocities are marked as circles.

\section{Appendix \\ Estimate of Primary Mass Lower Bound}

Given that all stars in our sample are known to be M dwarfs from their spectral information, we know that any calculated lower limit on the mass of the primary component must not fall above about $0.5 M_{\odot}$. This calculation acts as an independent check on the orbital fit, which is useful given the solution degeneracy that is prevalent along the period axis.

Isolate $P^{2}$ from Newton's formulation of Kepler's third law,

$$
P^{2}=(2 \pi)^{2} \frac{a^{3}}{G M_{\text {prim }}(1+q)},
$$

and an approximate expression of mean orbital speed,

$$
v_{o} \approx \frac{2 \pi a}{P}\left[1-\frac{1}{4} e^{2}-\frac{3}{64} e^{4}\right]
$$

where $v_{o} \approx K$. This approximation is sufficiently accurate for low $e$ and pushes the estimate down for nonzero $e$. Edge-on orientation is assumed, firmly establishing this calculation as a lower bound on primary mass:

$$
K \leqslant \frac{2 \pi a}{P}\left[1-\frac{1}{4} e^{2}-\frac{3}{64} e^{4}\right] .
$$

Substitution and simplification ultimately give

$$
M_{\text {prim }} \geqslant \frac{K^{3} P}{2 \pi G(1+q)\left[1-\frac{1}{4} e^{2}-\frac{3}{64} e^{4}\right]^{3}} .
$$

If the orbital solution places this value significantly above $0.5 M_{\odot}$, then it cannot be a correct solution.

\section{ORCID iDs}

Kevin R. Covey (1) https://orcid.org/0000-0001-6914-7797 Chad F. Bender (1) https://orcid.org/0000-0003-4384-7220 Nathan De Lee (1) https://orcid.org/0000-0002-3657-0705

Carles Badenes (i) https://orcid.org/0000-0003-3494-343X Dmitry Bizyaev (10 https://orcid.org/0000-0002-3601-133X Cullen H. Blake (i) https://orcid.org/0000-0002-6096-1749 Adam Burgasser (ib https://orcid.org/0000-0002-6523-9536 Joleen Carlberg (i) https://orcid.org/0000-0001-5926-4471 Scott W. Fleming (1) https://orcid.org/0000-0003-0556-027X 
D. A. García-Hernández (1) https://orcid.org/0000-00021693-2721

Marina Kounkel (1) https://orcid.org/0000-0002-5365-1267

Suvrath Mahadevan (1) https://orcid.org/0000-00019596-7983

Steven R. Majewski iㅏ https://orcid.org/0000-0003-

2025-3147

Dante Minniti (1) https://orcid.org/0000-0002-7064-099X

David Nidever (1) https://orcid.org/0000-0002-1793-3689

Kaike Pan (i) https://orcid.org/0000-0002-2835-2556

Keivan Stassun (i) https://orcid.org/0000-0002-3481-9052

Ryan Terrien (1i) https://orcid.org/0000-0002-4788-8858

\section{References}

Abrams, M. C., Davis, S. P., Rao, M. L. P., Engleman, R., Jr., \& Brault, J. W. 1994, ApJS, 93, 351

Albareti, F. D., Allende Prieto, C., Almeida, A., et al. 2017, ApJS, 233, 25

Allard, F., Homeier, D., Freytag, B., \& Sharp, C. M. 2012, in EAS Publications Series, Vol. 57, EAS Publications Series, ed. C. Reylé, C. Charbonnel, \& M. Schultheis (Les Ulis: EDP Sciences), 3

Bate, M. R., Bonnell, I. A., \& Bromm, V. 2002, MNRAS, 336, 705

Bender, C., Simon, M., Prato, L., Mazeh, T., \& Zucker, S. 2005, AJ, 129, 402

Bender, C. F., Mahadevan, S., Deshpande, R., et al. 2012, ApJL, 751, L31

Burgasser, A. J., Kirkpatrick, J. D., Cruz, K. L., et al. 2006, ApJS, 166, 585

Ciardi, D. R., von Braun, K., Bryden, G., et al. 2011, AJ, 141, 108

Claret, A. 2000, A\&A, 363, 1081

Clark, B. M., Blake, C. H., \& Knapp, G. R. 2012, ApJ, 744, 119

Clough, S. A., Shephard, M. W., Mlawer, E. J., et al. 2005, JQSRT, 91, 233

Cushing, M. C., Vacca, W. D., \& Rayner, J. T. 2004, PASP, 116, 362

Delfosse, X., Forveille, T., Ségransan, D., et al. 2000, A\&A, 364, 217

Deshpande, R., Blake, C. H., Bender, C. F., et al. 2013, AJ, 146, 156

Duchêne, G., \& Kraus, A. 2013, ARA\&A, 51, 269

Eisenstein, D. J., Weinberg, D. H., Agol, E., et al. 2011, AJ, 142, 72

El-Badry, K., Rix, H.-W., Ting, Y.-S., et al. 2018a, MNRAS, 473, 5043

El-Badry, K., Ting, Y.-S., Rix, H.-W., et al. 2018b, MNRAS, 476, 528

Fabrycky, D., \& Tremaine, S. 2007, ApJ, 669, 1298

Fernandez, M. A., Covey, K. R., De Lee, N., et al. 2017, PASP, 129, 084201

Fischer, D. A., \& Marcy, G. W. 1992, ApJ, 396, 178

Foreman-Mackey, D., Hogg, D. W., Lang, D., \& Goodman, J. 2013, PASP, 125,306

García Pérez, A. E., Allende Prieto, C., Holtzman, J. A., et al. 2016, AJ, 151,144

Gilhool, S. H., Blake, C. H., Terrien, R. C., et al. 2018, AJ, 155, 38

Goodman, J., \& Weare, J. 2010, CAMCS, 5, 65
Gorti, U., \& Bhatt, H. C. 1996, MNRAS, 283, 566

Grieves, N., Ge, J., Thomas, N., et al. 2017, MNRAS, 467, 4264

Gunn, J. E., Siegmund, W. A., Mannery, E. J., et al. 2006, AJ, 131, 2332

Holtzman, J. A., Shetrone, M., Johnson, J. A., et al. 2015, AJ, 150, 148

Jenkins, J. S., Ramsey, L. W., Jones, H. R. A., et al. 2009, ApJ, 704, 975

Kiseleva, L. G., Eggleton, P. P., \& Mikkola, S. 1998, MNRAS, 300, 292

Kratter, K. M. 2011, in ASP Conf. Ser. 447, Evolution of Compact Binaries, ed. L. Schmidtobreick, M. R. Schreiber, \& C. Tappert (San Francisco, CA: ASP), 47

Kraus, A. L., \& Hillenbrand, L. A. 2007, AJ, 134, 2340

Lépine, S., \& Gaidos, E. 2011, AJ, 142, 138

Lépine, S., \& Shara, M. M. 2005, AJ, 129, 1483

Majewski, S. R., Schiavon, R. P., Frinchaboy, P. M., et al. 2017, AJ, 154, 94

Mann, A. W., Feiden, G. A., Gaidos, E., Boyajian, T., \& von Braun, K. 2015, ApJ, 804, 64

Moe, M., \& Di Stefano, R. 2017, ApJS, 230, 15

Murphy, M. T., Tzanavaris, P., Webb, J. K., \& Lovis, C. 2007, MNRAS, 378, 221

Nidever, D. L., Holtzman, J. A., Allende Prieto, C., et al. 2015, AJ, 150, 173

Nutzman, P., \& Charbonneau, D. 2008, PASP, 120, 317

Offner, S. S. R., Kratter, K. M., Matzner, C. D., Krumholz, M. R., \& Klein, R. I. 2010, ApJ, 725, 1485

Pourbaix, D., Tokovinin, A. A., Batten, A. H., et al. 2004, A\&A, 424, 727

Qi, Z., Yu, Y., Bucciarelli, B., et al. 2015, AJ, 150, 137

Raghavan, D., McAlister, H. A., Henry, T. J., et al. 2010, ApJS, 190, 1

Ramsey, L. W., Adams, M. T., Barnes, T. G., et al. 1998, Proc. SPIE, 3352 , 34

Reipurth, B., \& Clarke, C. 2001, AJ, 122, 432

Shan, Y., Johnson, J. A., \& Morton, T. D. 2015, ApJ, 813, 75

Shetrone, M., Cornell, M. E., Fowler, J. R., et al. 2007, PASP, 119, 556

Souto, D., Cunha, K., García-Hernández, D. A., et al. 2017, ApJ, 835, 239

Tohline, J. E. 2002, ARA\&A, 40, 349

Tonry, J., \& Davis, M. 1979, AJ, 84, 1511

Tull, R. G. 1998, Proc. SPIE, 3355, 387

Viaux, N., Catelan, M., Stetson, P., et al. 2013, yCat, 355, 0

Walkowicz, L. M., Basri, G., Batalha, N., et al. 2011, AJ, 141, 50

Ward-Duong, K., Patience, J., De Rosa, R. J., et al. 2015, MNRAS, 449, 2618

Wilson, J. C., Hearty, F., Skrutskie, M. F., et al. 2010, Proc. SPIE, 7735, $77351 \mathrm{C}$

Wilson, J. C., Hearty, F., Skrutskie, M. F., et al. 2012, Proc. SPIE, 8446 , $84460 \mathrm{H}$

Wilson, O. C. 1941, ApJ, 93, 29

Zacharias, N., Finch, C., \& Frouard, J. 2017, AJ, 153, 166

Zacharias, N., Finch, C. T., Girard, T. M., et al. 2013, AJ, 145, 44

Zacharias, N., Monet, D. G., Levine, S. E., et al. 2005, yCat, 1297, 0

Zasowski, G., Johnson, J. A., Frinchaboy, P. M., et al. 2013, AJ, 146, 81

Zucker, S., \& Mazeh, T. 1994, ApJ, 420, 806

Zucker, S., Torres, G., \& Mazeh, T. 1995, ApJ, 452, 863 